\title{
General Relativistic Shock Waves that Extend the Oppenheimer-Snyder Model
}

\author{
Joel Smoller \& Blake Temple
}

Communicated by T.-P. LiU

\begin{abstract}
In earlier work we constructed a class of spherically symmetric, fluid dynamical shock waves that satisfy the Einstein equations of general relativity. These shock waves extend the celebrated Oppenheimer-Snyder result to the case of non-zero pressure. Our shock waves are determined by a system of ordinary differential equations that describe the matching of a FriedmannRobertson-Walker metric (a cosmological model for the expanding universe) to an Oppenheimer-Tolman metric (a model for the interior of a star) across a shock interface. In this paper we derive an alternate version of these ordinary differential equations, which are used to demonstrate that our theory generates a large class of physically meaningful (Lax-admissible) outgoing shock waves that model blast waves in a general relativistic setting. We also obtain formulas for the shock speed and other important quantities that evolve according to the equations. The resulting formulas are important for the numerical simulation of these solutions.
\end{abstract}

\section{Introduction}

In [7] we constructed a class of physically interesting shock-wave solutions of the Einstein equations of general relativity, and in [8] we applied these results to construct an exact solution of these equations that models an explosion into a static, singular, isothermal sphere. This solution provides a general relativistic model that parallels a Newtonian model for stellar formation. The paper [7] concluded with the derivation of a set of ordinary differential equations that describe the matching of a Friedmann-RobertsonWalker (FRW) type metric, to an Oppenheimer-Tolman (OT) type metric, such that the interface between the two metrics defines a spherically symmetric, fluid dynamical shock wave. 
In this paper we derive an alternative, simpler version of these ordinary differential equations (cf. Theorem 7 below), and we use these ordinary differential equations to compute simplified formulas for the physical quantities (the pressure, the density, and the sound, shock and characteristic speeds) that are determined by the equations. When computing the sound speeds we make the assumption that the equations of state are of the simple form $p=p(\rho)$, so that the sound speed is given by $d p / d \rho$. We apply these formulas to obtain conditions under which the Lax shock conditions hold, conditions under which the pressure is greater behind the shock, and conditions under which all "speeds" are bounded by the speed of light $c=1$; cf. [4]. In this paper we restrict our attention to the case when the shock wave lies outside of the Schwarzschild radius $(A>0 ;$ cf. (2.21) below). The ordinary differential equations and formulas we derive here can be used effectively in the numerical simulation of these shock-wave solutions.

The FRW metric is a uniformly expanding (or contracting) solution of the Einstein gravitational field equations and is generally accepted as a cosmological model for the expanding universe. The OT solution is a time-independent solution which models the interior of a star. Both metrics are spherically symmetric, and both are determined by a system of ordinary differential equations that close when an equation of state $p=p(\rho)$ for the fluid is specified. In our shock-wave solution, the FRW metric is an exploding inner core (of a star or the universe as a whole), and the boundary of this inner core is a shock surface that is driven by the expansion behind the shock into the outer, static, OT solution, which we imagine as the outer layers of a star, or the outer regions of the universe. (In the exact solution constructed in [8], the shock wave emerges from $\bar{r}=0$ at the initial (big-bang) singularity in the FRW metric, and thus the model provides a scenario by which the bigbang begins with a shock-wave explosion.)

In [7], we described a general procedure for matching different metric solutions of the Einstein equations across an interface such that the metrics match Lipschitz continuously at the interface. In order for the interface to be a true fluid dynamical shock wave (as opposed to a surface layer), we must impose an additional constraint, called the conservation constraint, on the equations. In [7], we showed that for the matching of a FRW metric to an OT metric, the Lipschitz continuous matching at an interface can be achieved with any two arbitrary equations of state assigned to the FRW and OT solutions separately. However, in order to satisfy the conservation constraint, we must impose one additional constraint, and thus we lose the freedom to impose one of the two equations of state. Said differently, the conservation constraint determines the inner FRW equation of state from the outer OT solution. Therefore, for any given fixed OT metric, our ordinary differential equations (cf. (3.20)-(3.23) below) describe the evolution of the shock position, together with the density, pressure and cosmological scale factor of the FRW solution. This FRW metric (which satisfies the ordinary differential equations) matches the given OT metric Lipschitz continuously across a true fluid dynamical shock wave. (This matching can 
be improved to a $C^{1,1}$ matching via a coordinate transformation; cf. Theorem 2 below.)

It is well known that the FRW metric exhibits qualitatively different behavior depending on the sign of $k$ (a parameter in the metric [11]), which determines the sign of the scalar curvature on the constant curvature surfaces at each fixed time. When $k>0$, the shock-wave solutions described in [7] reduce to the well-known model of OpPenheImer \& SNYDER (OS) when the OT solution is taken to be the empty space Schwarzschild metric. In this case the general ordinary differential equations derived in [7] reproduce the OS equation of state $p \equiv 0$ in the FRW metric, and thus our ordinary differential equations reproduce the OS results in this limit. Thus our shock-wave solutions provide a natural generalization of the OS model to the case of non-zero pressure. However, there is an important difference between the OS solution and our shock-wave solutions, namely, the OS interface is a time-reversible contact discontinuity, but the interfaces in our model describe true, timeirreversible, fluid dynamical, shock waves. Indeed, for a contact discontinuity, a smooth regularization of the solution at a fixed time propogates as a nearby smooth solution for all times thereafter. In contrast, it is well known from the theory of hyperbolic conservation laws that, due to time-irreversibility, shock-wave solutions cannot be approximated globally by smooth, shockfree solutions of the hyperbolic equations $[4,5]$.

The plan of the paper is as follows. In Section 2 we summarize the results in $[7,8]$. In Section 3 we analyze the conservation constraint, and obtain formulas for the FRW pressure. We prove that when the FRW density is greater than the OT density at the shock (the case of an explosion when the FRW metric is placed inside the OT metric), the FRW pressure is positive if and only if it is greater than the OT pressure. In Section 4, we use our formulas for the pressure to obtain formulas for the shock speed and fluid speed, and we discuss the Lax shock conditions. We also show that for sufficiently strong shocks, the equation of state can be approximated to first order in a neighborhood of a given point on both sides of the shock. In Section 5, we obtain formulas for the FRW sound speed; this is required for the verification of the Lax shock conditions. In Section 6 we show that outside the Schwarzschild radius, the FRW density must exceed the OT density at the shock in order that the sound speed be positive. This implies that our solutions model explosions, but not gravitational collapse (except in the Oppenheimer-Snyder limit $p=0$ ).

\section{Preliminaries}

In this section we review the results in [7] and [8]. We consider the Einstein gravitational field equations

$$
G=\kappa T,
$$

where $G$ denotes the Einstein curvature tensor for the space-time metric $g, T$ denotes the stress-energy tensor for a perfect fluid: 


$$
T=(\rho+p) u \otimes u+p g,
$$

and $\kappa=8 \pi \mathscr{G}$. (We assume that the speed of light $c=1$.) Here $u$ is the 4-velocity of the fluid, $\mathscr{G}$ is Newton's gravitational constant, and we assume a barotropic equation of state of the form $p=p(\rho)$, where $p$ is the pressure and $\rho$ is the density. In a given coordinate system, $T$ takes the form

$$
T_{i j}=p g_{i j}+(p+\rho) u_{i} u_{j},
$$

where $i, j$ are assumed to run from 0 to 3, and we use the Einstein summation convention throughout. The Einstein tensor $G$ is constructed from the Riemann curvature tensor so as to satisfy $\operatorname{div} G=0$. Thus, on solutions of (2.1), $\operatorname{div} T=0$, and this is the relativistic version of the classical Euler equations for compressible fluid flow. The Euler equations for compressible fluids provide the setting for the mathematical theory of shock waves [4]. We now briefly recall the FRW and OT metrics, and the results of [7].

The FRW metric describes a spherically symmetric space-time that is homogeneous and maximally symmetric at each fixed time. In coordinates, the FRW metric is given by [11]

$$
d s^{2}=-d t^{2}+R^{2}(t)\left\{\frac{1}{1-k r^{2}} d r^{2}+r^{2} d \Omega^{2}\right\},
$$

where $t \equiv x^{0}, r \equiv x^{1}, \theta \equiv x^{2}, \varphi \equiv x^{3}, R \equiv R(t)$ is the 'cosmological scale factor', and $d \Omega^{2}=d \theta^{2}+\sin ^{2} \theta d \varphi^{2}$ denotes the standard metric on the unit 2-sphere. The constant $k$ can be normalized to be either $+1,-1$, or 0 by appropriately rescaling the radial variable, and each of the three cases is qualitatively different. We assume that the fluid is perfect (i.e., (2.3) holds), and that the fluid is co-moving with the metric. The fluid is said to be comoving relative to a background metric $g_{i j}$ if $u^{i}=0, i=1,2,3$, so that $g$ being diagonal and $u$ having length 1 imply [11] that

$$
u^{0}=\sqrt{-g_{00}} .
$$

Substituting (2.4) into the field equations, and making the assumption that the fluid is perfect and co-moving with the metric, yields the following constraints on the unknown functions $R(t), \rho(t)$ and $p(t)[11,7]$ :

$$
\begin{gathered}
3 \ddot{R}=-4 \pi \mathscr{G}(\rho+3 p) R, \\
R \ddot{R}+2 \dot{R}^{2}+2 k=4 \pi \mathscr{G}(\rho-p) R^{2},
\end{gathered}
$$

together with

$$
\dot{p} R^{3}=\frac{d}{d t}\left\{R^{3}(p+\rho)\right\} .
$$

Equation (2.8) is equivalent to

$$
p=-\rho-\frac{R \dot{\rho}}{3 \dot{R}} .
$$


Substituting (2.6) into (2.7) we get

$$
\dot{R}^{2}+k=\frac{8 \pi \mathscr{G}}{3} \rho R^{2}
$$

Since $\rho$ and $p$ are assumed to be functions of $t$ alone in (2.4), equations (2.9) and (2.10) give two equations for the two unknowns $R$ and $\rho$ under the assumption that the equation of state is of the form $p=p(\rho)$. It follows from (2.9), (2.10) (cf. [7]) that $(R(t), \rho(t))$ is a solution if and only if $(R(-t), \rho(-t))$ is a solution, and that

$$
\dot{\rho} \dot{R}<0 .
$$

Thus to every expanding solution there exists a corresponding contracting solution, and conversely.

The OT metric describes a time-independent, spherically symmetric solution that models the interior of a star. In coordinates the components of the metric are given by

$$
d \bar{s}^{2}=-B(\bar{r}) d \bar{t}^{2}+A(\bar{r})^{-1} d \bar{r}^{2}+\bar{r}^{2} d \Omega^{2} .
$$

We write this metric in bar-coordinates so that it can be distinguished from the unbarred coordinates when the metrics are matched. Assuming that the stress tensor is that of a perfect fluid which is co-moving with the metric, and substituting (2.12) into the field equations (2.1) yields (cf. [11])

$$
A(\bar{r})=\left(1-\frac{2 \mathscr{G} M}{\bar{r}}\right),
$$

where $M \equiv M(\bar{r}), \bar{\rho} \equiv \bar{\rho}(\bar{r})$ and $\bar{p} \equiv \bar{p}(\bar{r})$ satisfy the following system of ordinary differential equations in the unknown functions $(\bar{\rho}(\bar{r}), p(\bar{r}), M(\bar{r}))$ :

$$
\begin{gathered}
\frac{d M}{d \bar{r}}=4 \pi \bar{r}^{2} \bar{\rho} \\
-\bar{r}^{2} \frac{d \bar{p}}{d \bar{r}}=\mathscr{G} M \bar{\rho}\left\{1+\frac{\bar{p}}{\bar{\rho}}\right\}\left\{1+\frac{4 \pi \bar{r}^{3} \bar{p}}{M}\right\}\left\{1-\frac{2 \mathscr{G} M}{\bar{r}}\right\}^{-1} .
\end{gathered}
$$

Equation (2.15) is called the Oppenheimer-Volkov equation, and is referred to by WEINBERG as the fundamental equation of Newtonian astrophysics with general-relativistic corrections supplied by the last three terms [11, page 301].

In this paper we assume the case of a barotropic equation of state $\bar{p}=\bar{p}(\bar{\rho})$, in which case equations $(2.14),(2.15)$ yield a system of two ordinary differential equations in the two unknowns $(\bar{\rho}, M)$. We always assume that

$$
0<\frac{\bar{p}}{\bar{\rho}} \equiv \bar{\mu}<1
$$

and that the sound speed is less than the (normalized) speed of light; i.e., 


$$
0<\bar{\sigma} \equiv \frac{d \bar{p}}{d \bar{\rho}} \leqq 1
$$

The total mass $M$ inside radius $\bar{r}$ is then defined by

$$
M(\bar{r})=\int_{0}^{\bar{r}} 4 \pi \xi^{2} \bar{\rho}(\xi) d \xi .
$$

The metric component $B \equiv B(\bar{r})$ is determined from $\bar{\rho}$ and $M$ through the equation

$$
\frac{B^{\prime}(\bar{r})}{B}=-2 \frac{\bar{p}^{\prime}(\bar{r})}{\bar{p}+\bar{\rho}}
$$

We remark that for any given FRW and OT metrics, there are maximal domains of definitions for the variables. We assume that the FRW metric is defined on the maximal interval $t_{-}<t<t_{+}$and $0 \leqq r_{-}<r<r_{+}$, and that the OT metric is defined on the maximal interval $0<\bar{r}_{-}<\bar{r}<\bar{r}_{+}$. For example, if $k>0$, then we must have $r<1 / \sqrt{k}, t$ must be restricted so that $\rho(t)$ and $R(t)$ are positive, and by (2.10), we must require that $\frac{8 \pi}{3} \mathscr{G} \rho(t)$ $R(t)^{2}-k \geqq 0$.

In [7], we described a procedure for constructing a coordinate transformation $(\bar{t}, \bar{r}) \rightarrow(t, r)$ such that the FRW metric (2.4) matches the OT metric (2.12) Lipschitz continuously across a shock surface $\Sigma$. This shock surface is given implicitly by the equation

$$
M(\bar{r})=\frac{4 \pi}{3} \rho(t) \bar{r}^{3} .
$$

Equation (2.18) defines the radial coordinate $\bar{r}$ of the OT metric as a function of the time coordinate $t$ of the FRW metric along the shock surface $\Sigma$. Note that for a given FRW density $\rho(t),(2.18)$ determines $\bar{r}=\bar{r}(t)$, the shock position. However, we can also solve (2.18) for $\rho$ to obtain $\rho$ as a function of $\bar{r}$, namely,

$$
\rho=\frac{3}{4 \pi} \frac{M(\bar{r})}{\bar{r}^{3}} .
$$

From here on we let $\rho$ refer to either $\rho(t)$ or $\rho(\bar{r})$, as given by (2.18), so that (with slight abuse of notation)

$$
\rho(t)=\rho(\bar{r}(t)),
$$

on the shock surface. With this notation, $\rho(\bar{r})$ is determined by the OT solution alone.

For (2.18) to be meaningful in a given problem we must assume that $\bar{r}=\bar{r}(t)$ is defined for $t \in\left(t_{-}, t_{+}\right), \bar{r} \in\left(\bar{r}_{-}, \bar{r}_{+}\right)$, and $r=\frac{\bar{r}(t)}{R(t)} \in\left(r_{-}, r_{+}\right)$. Equation (2.10) applies when any equation of state $p=p(\rho)$ is assigned to the FRW metric, and any equation of state $\bar{p}=\bar{p}(\bar{\rho})$ is assigned to the OT metric. The transformation $\bar{r}=\bar{r}(t, r)$ is given by 


$$
\bar{r}=R(t) r
$$

in the mapping $(\bar{t}, \bar{r}) \rightarrow(t, r)$, but the transformation $\bar{t}=\bar{t}(t, r)$ is more complicated, and its existence is demonstrated in [7]. It is somewhat remarkable that, other than its existence, for our developments, we do not require any detailed information about the $\bar{t}$ transformation.

The following result summarizes the result in Section 4 of [7, pp. 280 285].

Theorem 1. Let any FRW and OT metrics be given such that the shock surface $\bar{r}=\bar{r}(t)$ is defined implicitly by (2.18) in a neighborhood of a point $\left(t_{0}, \bar{r}_{0}\right)$, $t_{-}<t_{0}<t_{+}, \bar{r}_{-}<\bar{r}<\bar{r}_{+}$, and $r_{-}<r \equiv \frac{\bar{r}}{R}<r_{+}$. Assume that

$$
\begin{gathered}
\frac{d \bar{p}}{d \bar{\rho}}>0, \\
A\left(\bar{r}_{0}\right)>0,
\end{gathered}
$$

and hence

$$
1-k r_{0}^{2}>0, \quad\left(r_{0}=\bar{r}_{0} / R\left(t_{0}\right)\right) .
$$

Then for any value of $\bar{t}_{0}$, there exists a mapping $(t, r) \rightarrow(\bar{t}, \bar{r})$ (defined in Section 4 of [7]), which is denoted by

$$
\Psi(t, r) \equiv(\bar{t}(t, r), \bar{r}(t, r)), \quad \bar{r}(t, r)=R(t) r,
$$

such that $\Psi$ is one-to-one and regular in a neighborhood of the point $\left(t_{0}, r_{0}\right),{ }^{1}$ and takes the open interval $\left(t_{0}, r_{0}\right)$ into the open interval $\left(\bar{t}_{0}, \bar{r}_{0}\right)$. Moreover, under this coordinate identification, the given FRW and OT metrics match Lipschitz continuously across the surface (2.18). The condition

$$
\bar{r}(t, r)=R(t) r=\bar{r}
$$

implies that the areas of the spheres of symmetry agree in the barred and unbarred frames, and the shock surface in $(t, r)$-coordinates is given by $r(t)=\bar{r}(t) / R(t)(\bar{r}(t)$ denotes the shock position as distinguished from the coordinate function $\bar{r}(t, r)$ defined in (2.24)).

Remarks. The condition (2.20) says that the OT sound speed is positive, condition (2.21) says that $\bar{r}$ is not at the "Schwarzschild radius", and condition (2.22) says that the value of $r_{0}$ is not outside the FRW universe, i.e., it is inside the region of validity of the FRW coordinate system.

Throughout the remainder of this paper we assume implicitly that (2.20)(2.22) hold on every Oppenheimer-Snyder-type shock wave that we discuss.

\footnotetext{
${ }^{1}$ Note that the mapping $(t, \bar{r}) \rightarrow(\bar{t}, \bar{r})$ is $1-1$ whenever the mapping $(t, r) \rightarrow(\bar{t}, \bar{r})$ is $1-1$, because the mapping $(t, r) \rightarrow(t, \bar{r}) \equiv(t, R(t) r)$ has Jacobian $R>0$.
} 
Our construction in Theorem 1 guarantees that the FRW metric matches the OT metric Lipschitz continuously across the shock (2.18), and thus the following general theorem (which is proved in [7, Theorem 4]; see also [1]) applies:

Theorem 2. Let $\Sigma$ denote a smooth, 3-dimensional shock surface in space-time with spacelike normal vector $\mathbf{n}$. Assume that the components $g_{i j}$ of the gravitational metric $g$ are smooth on either side of $\Sigma$ (continuous up to the boundary on either side separately), and Lipschitz continuous across $\Sigma$ in some fixed coordinate system. Then the following statements are equivalent:

(i) $[K]=0$ at each point of $\Sigma$. (Here, $[f]$ denotes the jump in the quantity $f$ across the surface $\Sigma$, and $K$ denotes the extrinsic curvature, or second fundamental form, which is determined by $g$ separately on each side of the shock surface $\Sigma$.)

(ii) The curvature tensors $R_{j k l}^{i}$ and $G_{i j}$, viewed as second-order operators on the metric components $g_{i j}$, produce no delta function sources on $\Sigma$.

(iii) For each point $P \in \Sigma$ there exists a $C^{1,1}$ coordinate transformation defined in a neighborhood of $P$, such that, in the new coordinates, (which can be taken to be the Gaussian normal coordinates for the surface), the metric components are $C^{1,1}$ functions of these coordinates. (By $C^{1,1}$ we mean that the first derivatives are Lipschitz continuous.)

(iv) For each $P \in \Sigma$, there exists a coordinate frame that is locally Lorentzian at $P$, and can be obtained from the original coordinates by a $C^{1,1}$ coordinate transformation. (A coordinate frame is locally Lorentzian at a point $P$ if $g_{i j}(P)=\operatorname{diag}(-1,1,1,1)$ and $g_{i j, k}(P)=0$ for all $i, j, k=0, \ldots, 3$.)

Moreover, if any one of these equivalencies hold, then the Rankine-Hugoniot jump conditions $\left[G^{i j}\right] n_{i}=0$ hold at each point on $\Sigma$. (This expresses the weak form of conservation of energy and momentum across $\Sigma$ when $G=\kappa T$.)

In the case of spherical symmetry, the conservation condition $\left[G^{i j}\right] n_{i}=0$ reduces to one condition $\left[G^{i j}\right] n_{i} n_{j}=0$, and this is what implies the equivalencies in Theorem 1. In fact, we have [7, Proposition 9]:

Theorem 3. Assume that $g$ and $\bar{g}$ are two spherically symmetric metrics that match Lipschitz continuously across a three-dimensional shock interface $\Sigma$ to form the matched metric $g \cup \bar{g}$. That is, assume that $g$ and $\bar{g}$ are Lorentzian metrics given by

$$
\begin{aligned}
& d s^{2}=-a(t, r) d t^{2}+b(t, r) d r^{2}+c(t, r) d \Omega^{2}, \\
& d \bar{s}^{2}=-\bar{a}(\bar{t}, \bar{r}) d \bar{t}^{2}+\bar{b}(\bar{t}, \bar{r}) d \bar{r}^{2}+\bar{c}(\bar{t}, \bar{r}) d \Omega^{2},
\end{aligned}
$$

and that there exists a smooth coordinate transformation $\Psi:(t, r) \rightarrow(\bar{t}, \bar{r})$, defined in a neighborhood of a shock surface $\Sigma$ given by $r=r(t)$ such that the metrics agree on $\Sigma$. (We implicitly assume that $\theta$ and $\varphi$ are continuous across the surface.) Assume that 


$$
c(t, r)=\bar{c}(\Psi(t, r))
$$

in an open neighborhood of the shock surface $\Sigma$, so that, in particular, the areas of the 2-spheres of symmetry in the barred and unbarred metrics agree on the shock surface. Assume also that the shock surface $r=r(t)$ in unbarred coordinates is mapped to the surface $\bar{r}=\bar{r}(\bar{t})$ by $(\bar{t}, \bar{r}(\bar{t}))=\Psi(t, r(t))$. Assume, finally, that the normal $\mathbf{n}$ to $\Sigma$ is non-null, and that $\mathbf{n}(c) \neq 0$ where $\mathbf{n}(c)$ denotes the derivative of the function $c$ in the direction of the vector $\mathbf{n}$. Then the following are equivalent to the statement that the components of the metric $g \cup \bar{g}$ in any Gaussian normal coordinate system are $C^{1,1}$ functions of these coordinates across the surface $\Sigma$ :

$$
\begin{aligned}
{\left[G_{j}^{i}\right] n_{i} } & =0, \\
{\left[G^{i j}\right] n_{i} n_{j} } & =0, \\
{[K] } & =0 .
\end{aligned}
$$

Here, $[f]=\bar{f}-f$ denotes the jump in the quantity $f$ across $\Sigma$, and, as before, $K$ denotes the second fundamental form on the shock interface.

It is straightforward to check that the conditions in Theorem 3 on the functions $c$ and $\bar{c}$ are met when $\bar{c}=\bar{r}, c=R r$, and $\bar{r}(t, r)=R(t) r$. In light of (2.25) and (2.26), we conclude that conservation across the shock surface (2.18) is equivalent to the condition that the equation $\left[T^{i j}\right] n_{i} n_{j}=0$ holds across $\Sigma$. In [7] we derived the identity

$$
\left[T^{i j}\right] n_{i} n_{j}=(\rho+p) n_{0}^{2}-(\bar{\rho}+\bar{p}) \frac{\bar{n}_{0}^{2}}{B}+(p-\bar{p})|\mathbf{n}|^{2} .
$$

Here $n^{i}$ and $\bar{n}^{i}$ denote the components of the normal vector $\mathbf{n}$ to $\Sigma$ in the $(t, r)$ and $(\bar{t}, \bar{r})$ coordinate systems, respectively. Equation (2.28) represents the additional constraint (the "conservation constraint") imposed by conservation across the shock surface (2.18). Using the expressions for the components $n_{i}$ and $\bar{n}_{i}$ of $\mathbf{n}$, we readily obtain the following equivalent expression which we refer to as the "constraint equation" (see (5.34) of [7])

$$
\left[T^{i j}\right] n_{i} n_{j}=(\bar{p}+\rho) \dot{r}^{2}-(\bar{\rho}+\bar{p}) \frac{\left(1-k r^{2}\right)}{A R^{2}} \dot{\bar{r}}^{2}+(p-\bar{p}) \frac{1-k r^{2}}{R^{2}}=0 .
$$

Here, $\dot{r}, \dot{\bar{r}}$ denote the shock speeds $\frac{d r}{d t}, \frac{d \bar{r}}{d t}$, respectively. In [7], we used equation (2.9) to eliminate $p$ from (2.29), and thereby derived an autonomous system of ordinary differential equations in $(R, r)$ as a function of $t$ that determine the inner FRW metric and the shock position $r(t)$ in terms of any given OT metric (cf. (5.46)-(5.49) of [7]). Thus, for any assignment of equation of state $\bar{p}=\bar{p}(\bar{\rho})$ and initial conditions for an OT metric, our system of ordinary differential equations determines the FRW functions $R(t), \rho(t)$ and $p(t)$ that match the given OT metric Lipschitz continuously across the shock surface (2.18), such that conservation holds across the surface.

We derived in [8] an equivalent form of (2.29), namely, 
$0=(1-\theta)(\rho+\bar{p})(p+\bar{\rho})^{2}+(1-1 / \theta)(\bar{\rho}+\bar{p})(\rho+p)^{2}+(p-\bar{p})(\rho-\bar{\rho})^{2}$,

where

$$
\theta=\frac{A}{1-k r^{2}} .
$$

This form of the constraint equation enabled us to construct the exact solution in [8]. The development to follow is likewise based on an analysis of (2.30). For completeness, we include the derivation of (2.30) here, but before giving this we first show that $\theta \leqq 1$ is a natural condition. Indeed, by (2.10), the values of $R$ for which the FRW metric is defined must satisfy

$$
\dot{R}^{2}=\frac{8 \pi}{3} \mathscr{G} \rho R^{2}-k \geqq 0 .
$$

Using the shock surface equation (2.18), we can simplify this:

$$
\dot{R}^{2}=\frac{8 \pi}{3} \mathscr{G} \rho R^{2}-k=\frac{1}{r^{2}}\left\{\frac{2 G M}{\bar{r}}-k r^{2}\right\},
$$

and so

$$
\dot{R}^{2} r^{2}=-A+\left(1-k r^{2}\right) .
$$

This can be written as

$$
\dot{R}^{2} r^{2}=\left(1-k r^{2}\right)(1-\theta) .
$$

Thus the condition that

$$
0<\theta \leqq 1
$$

is equivalent to (2.32), in view of our assumptions (2.21) and (2.22). Moreover, since we are assuming that (2.21), (2.22) hold throughout, it is clear that (2.36) is equivalent to (2.32) when $k \leqq 0$ as well. In this paper we always assume that (2.36) holds.

We now give the derivation of (2.30). Differentiating (2.18) with respect to $t$ and applying (2.14) yields

$$
\dot{\rho}=\frac{3}{\bar{r}}(\bar{\rho}-\rho) \dot{\bar{r}}
$$

Solving for $\dot{\rho}$ in (2.9) yields

$$
\dot{\rho}=-\frac{3 \dot{R}}{R}(\rho+p) .
$$

Combining (2.37) and (2.38) thus gives

$$
\dot{\bar{r}}=\dot{R} r \frac{(\rho+p)}{(\rho-\bar{\rho})} .
$$


Differentiating $\bar{r}=R r$ with respect to $t$, using (2.39), and solving for $\dot{r}$ we get

$$
\dot{r}=\frac{\dot{R} r}{R} \frac{(\bar{\rho}+p)}{(\rho-\bar{\rho})} .
$$

Substituting (2.39) and (2.40) into (2.29), we obtain the following equation, which is equivalent to the conservation condition $\left[T^{i j}\right] n_{i} n_{j}=0$ :

$0=\left(\frac{1}{1-k r^{2}}\right)(\rho+\bar{p})(p+\bar{\rho})^{2}-\frac{1}{A}(\bar{\rho}+\bar{p})(\rho+p)^{2}+\frac{1}{r^{2} \dot{R}^{2}}(p-\bar{p})(\rho-\bar{\rho})^{2}$.

Equation (2.41) expresses conservation at the shock surface (2.18). But by (2.34),

$$
\dot{R}^{2} r^{2}=-A+\left(1-k r^{2}\right)
$$

holds on the shock surface, and using this we can transform (2.41) into the final form (2.30).

In the next section we develop a general theory of shock waves that extend the Oppenheimer-Snyder model, and our analysis is based on a careful study of $(2.30)$.

\section{The conservation constraint}

In this section we analyze (2.30) in detail. For convenience, we summarize the results of Section 2 in the following theorem:

Theorem 4. Assume that FRW and OT metrics are given that match Lipschitz continuously across the shock surface (2.18) such that (2.20)-(2.22) hold. Then (i)-(iv) of Theorem 2 hold on the shock surface if and only if (2.30) holds on the shock-surface.

Now solving for $p$ in (2.30), we obtain the following formula for the FRW pressure $p$ :

$$
p \pm=\frac{\frac{1}{2}\left\{-(\bar{\rho}+\rho)^{2}+2(\theta-1) \bar{\rho} \bar{p}+2\left(\theta+\frac{1}{\theta}\right) \rho \bar{\rho}+2\left(\frac{1}{\theta}-1\right) \rho \bar{p} \pm S Q\right\}}{(1-\theta) \rho+\left(2-\theta-\frac{1}{\theta}\right) \bar{p}+\left(1-\frac{1}{\theta}\right) \bar{\rho}}
$$

where

$$
S Q=\left(6 \bar{\rho}^{2} \rho^{2}-4 \rho^{3} \bar{\rho}-4 \overline{\rho^{3}} \rho+\rho^{4}+\bar{\rho}^{4}\right)^{1 / 2}=(\rho-\bar{\rho})^{2} .
$$

Thus we conclude that every OT solution determines two possible FRW pressures through the conservation constraint. These implicitly determine FRW equations of state $p=p(\rho)$. Let 


$$
\sigma \equiv \frac{d p}{d \rho}
$$

denote the sound speed, and let

$$
\mu \equiv \frac{p}{\rho} .
$$

Now the terms in the numerator of (3.1) combine as follows:

$-(\bar{\rho}+\rho)^{2}+2(\theta+1 / \theta) \rho \bar{\rho} \pm(\rho-\bar{\rho})^{2}=-2(2-\theta-1 / \theta) \rho \bar{\rho}-\left\{2(\rho-\bar{\rho})^{2}\right\}_{-}$,

where we use the notation that the bracket \{\}$_{-}$is taken to be zero unless we take the minus sign in (3.1) (and the corresponding minus sign in (3.3)). Substituting (3.3) in (3.1) gives

$$
\begin{aligned}
p \pm & =\frac{(\theta-1) \bar{\rho} \bar{p}+\left(\frac{1}{\theta}-1\right) \rho \bar{p}-\left(2-\theta-\frac{1}{\theta}\right) \rho \bar{\rho}-\left\{(\rho-\bar{\rho})^{2}\right\}_{-}}{(1-\theta) \rho+\left(2-\theta-\frac{1}{\theta}\right) \bar{p}+\left(1-\frac{1}{\theta}\right) \bar{\rho}} \\
& =\frac{-(1-\theta)\{\bar{\rho} \bar{p}-\rho \bar{\rho}\}+\left(\frac{1}{\theta}-1\right)\{\rho \bar{p}+\rho \bar{\rho}\}-\left\{(\rho-\bar{\rho})^{2}\right\}_{-}}{(1-\theta)\{\rho+\bar{p}\}-\left(\frac{1}{\theta}-1\right)\{\bar{p}+\bar{\rho}\}} \\
& =\frac{-(1-\theta) \bar{\rho}(\rho+\bar{p})+\left(\frac{1}{\theta}-1\right) \rho(\bar{p}+\bar{\rho})-\left\{(\rho-\bar{\rho})^{2}\right\}_{-}}{(1-\theta)\{\rho+\bar{p}\}-\left(\frac{1}{\theta}-1\right)\{\bar{p}+\bar{\rho}\}}
\end{aligned}
$$

which upon multiplying the numerator and denominator by $\theta /(1-\theta)$ yields

$$
\begin{aligned}
& p_{+}=\frac{-\theta \bar{\rho}(\rho+\bar{p})+\rho(\bar{\rho}+\bar{p})}{\theta(\rho+\bar{p})-(\bar{\rho}+\bar{p})}, \\
& p_{-}=\frac{-\theta \bar{\rho}(\rho+\bar{p})+\rho(\bar{\rho}+\bar{p})-\left\{\frac{\theta}{1-\theta}(\rho-\bar{\rho})^{2}\right\}}{\theta(\rho+\bar{p})-(\bar{\rho}+\bar{p})} .
\end{aligned}
$$

We can further simplify $p_{-}$as follows. First, we can verify the identity

$-\theta \bar{\rho}(\rho+\bar{p})+\rho(\bar{\rho}+\bar{p})-\frac{\theta}{1-\theta}(\rho-\bar{\rho})^{2}=\frac{1}{1-\theta}\left(\theta-\frac{\bar{\rho}+\bar{p}}{\rho+\bar{p}}\right)\left(\theta-\frac{\rho}{\bar{\rho}}\right)(\rho+\bar{p}) \bar{\rho}$.

Substituting this into the numerator of (3.6) yields

$$
p_{-}=\frac{\left(\frac{1}{1-\theta}\right)\left(\theta-\frac{\bar{\rho}+\bar{p}}{\rho+\bar{p}}\right)\left(\theta-\frac{\rho}{\bar{\rho}}\right)(\rho+\bar{p}) \bar{\rho}}{(\rho+\bar{p})\left(\theta-\frac{\bar{\rho}+\bar{p}}{\rho+\bar{p}}\right)}=\frac{\theta \bar{\rho}-\rho}{1-\theta} .
$$

Thus, if we define the variable

$$
\Theta \equiv \gamma \theta
$$

where

$$
\gamma \equiv \frac{\rho+\bar{p}}{\bar{\rho}+\bar{p}}
$$


then the pressures $p_{+}$and $p_{-}$take the similar forms

$$
\begin{aligned}
& p_{+}=\frac{\Theta \bar{\rho}-\rho}{1-\Theta}, \\
& p_{-}=\frac{\theta \bar{\rho}-\rho}{1-\theta} .
\end{aligned}
$$

The following two theorems follow directly from (3.9). (In Section 5 we will prove that the case $\bar{\rho}>\rho$ leads to $\frac{d p}{d \rho}<0$, a non-physical sound speed.)

Theorem 5. Assume that (2.20)-(2.22) hold and that

$$
z \equiv \frac{\bar{\rho}}{\rho}<1, \quad \bar{\mu} \equiv \frac{\bar{p}}{\bar{\rho}} .
$$

Then $p_{+}>0$ if and only if $p_{+}-\bar{p}>0$ if and only if $\theta_{1} \leqq \theta<1$ at the shock, where

$$
\theta_{1} \equiv \theta_{1}(z, \bar{\mu}) \equiv \frac{1}{\gamma}=\frac{\bar{\rho}+\bar{p}}{\rho+\bar{p}}=\frac{1+\bar{\mu}}{1+\bar{\mu} z} z
$$

Theorem 6. Assume that (2.20)-(2.22) hold and that $z<1$. Then for every choice of positive values for $\bar{\rho}, \bar{p}$ and $\rho$, the pressure $p_{+}$monotonically takes on every value from $[\bar{p},+\infty)$, and the pressure difference $\left(p_{+}-\bar{p}\right)$ monotonically takes on every value from $[0,+\infty)$, as $\theta$ ranges monotonically from $\left[1, \theta_{1}\right)$.

Proof. When $\rho>\bar{\rho}$, it follows immediately from (3.8) and (3.9) that $p_{+}>0$ if and only if $\theta>\theta_{1}$. To see this, note that the numerator in (3.9) is always negative because

$$
\gamma \theta z=\frac{1+\bar{\mu} z}{1+\bar{\mu}} \gamma<1
$$

when $z<1$. Thus by (3.9), $p_{+}>0$ if and only if $\gamma \theta>1$. Furthermore, if $\bar{\rho}, \bar{p}$ and $\rho$ are fixed, then $p$ varies monotonically from $\bar{p}$ to $\infty$ as $\theta$ varies from +1 to $\theta_{1}$ because $p \theta<0$ (cf. (4.5) below), and when $\theta=1$,

$$
p_{+}=\frac{\frac{\rho+\bar{p}}{\bar{\rho}+\bar{p}} \bar{\rho}-\rho}{1-\frac{\rho+\bar{p}}{\bar{\rho}+\bar{p}}}=\bar{p} .
$$

We can perform a similar analysis on the difference $\left(p_{+}-\bar{p}\right)$, because, as is easily shown,

$$
p_{+}-\bar{p}=\left(\frac{1-\theta}{\gamma \theta-1}\right)(\bar{\rho}+\bar{p}) \gamma .
$$

This completes the proofs of the Theorems 5 and 6 .

Another direct consequence of (3.9), (3.10) is that if $A>0$ and $\theta<1$, then when $\rho>\bar{\rho}$, the only shock waves with positive pressure must satisfy $p=p_{+}$ and 


$$
\Theta \equiv \gamma \theta>1
$$

In this case (3.8) implies that

$$
\rho>\frac{1}{\theta} \rho+\left(1+\frac{1}{\theta}\right) \bar{p}
$$

Next, using the formulas (3.9) and (3.10) for $p_{-}$and $p_{+}$, we can now derive a simplified set of equations for the dynamics of the shock surface and the FRW metric, assuming a fixed OT metric satisfying (2.20)-(2.22), and assuming the conservation constraint holds. Differentiating (2.18) and using (2.14) gives

$$
\dot{\rho}=\frac{3(\bar{\rho}-\rho)}{\bar{r}} \dot{\bar{r}}
$$

Using (3.14) to substitute for $\dot{\rho}$ in (2.9) gives

$$
\dot{\bar{r}}=-\frac{p+\rho}{\bar{\rho}-\rho} \frac{\bar{r}}{R} \dot{R}
$$

Now using the formula (3.9) for $p_{+}$to substitute for $p$ in (3.15) we obtain (for $p_{-}$just set $\gamma=1$, or equivalently, substitute $\theta$ for $\Theta$ in the formulas that follow):

$$
\frac{p+\rho}{\bar{\rho}-\rho}=\frac{\frac{\Theta \bar{\rho}-\rho}{1-\Theta}+\rho}{\bar{\rho}-\rho}=\frac{\Theta}{1-\Theta} .
$$

Using this in (3.15) yields the first equation

$$
\dot{\bar{r}}=-\frac{\Theta}{1-\Theta} r \dot{R}
$$

We can get a correspondingly simple equation for $\dot{r}$ as follows: Using $r=\bar{r} / R$, we can differentiate with respect to $t$ and use (3.17) to obtain

$$
\dot{r}=\frac{1}{R}(\dot{\bar{r}}-r \dot{R})=\frac{1}{R}\left(-\frac{\Theta}{1-\Theta}-1\right) r \dot{R}
$$

which we write as

$$
\dot{r}=\frac{1}{R}\left(\frac{-1}{1-\Theta}\right) r \dot{R}
$$

Thus our system of equations in the case $p=p_{+}$can be taken as

$$
\begin{aligned}
r^{2} \dot{R}^{2} & =\left(1-k r^{2}\right)(1-\theta), \\
R \dot{r} & =\frac{-1}{1-\Theta} r \dot{R},
\end{aligned}
$$

where the choice of sign in (3.21) comes from the choice of square root when we solve for $\dot{R}$ in (3.20). Hence we can also write (3.20), (3.21) as

$$
r \dot{R}= \pm \sqrt{1-k r^{2}} \sqrt{1-\theta}
$$




$$
R \dot{r}=\mp \frac{1}{1-\Theta} \sqrt{1-k r^{2}} \sqrt{1-\theta}
$$

The equations when $p=p_{-}$are obtained by substituting $\theta$ for $\Theta$ in (3.23), namely,

$$
\begin{gathered}
r \dot{R}= \pm \sqrt{1-k r^{2}} \sqrt{1-\theta} \\
R \dot{r}=\mp \sqrt{\frac{1-k r^{2}}{1-\theta}} .
\end{gathered}
$$

Assuming that a fixed OT solution satisfying (2.20) is given, we can use equations (3.22), (3.23) and (3.24), (3.25) to obtain a set of autonomous ordinary differential equations for the shock position $r(t)$ and the cosmological scale factor $R(t)$ whose solutions determine the FRW metrics that match the given OT metric Lipschitz continuously across the shock surface (2.18) such that conservation holds across the shock. The solution is determined by the coordinate mapping (2.23) so long as (2.21) and (2.22) hold. To see this, note that fixing the OT metric directly determines $M(\bar{r}), A(\bar{r}), \bar{\rho}(\bar{r})$ and $\bar{p}(\bar{r})$, and we can use the shock surface condition to determine $\rho=\frac{3}{4 \pi} \frac{M(\bar{r})}{\bar{r}^{3}}$ as a known function of $\bar{r}$ as well. Since our coordinate identification sets $\bar{r}=R r$, all of these functions can be taken as known functions of the shock position $r(t)$ and scale factor $R(t)$. Thus

$$
\theta=\frac{A}{1-k r^{2}}
$$

is a known function of $(r(t), R(t))$, and

$$
\gamma \equiv \frac{\rho+\bar{\rho}}{\bar{\rho}+\bar{p}}
$$

is a known function of $(r(t), R(t))$, and hence

$$
\Theta=\gamma \theta
$$

is also a known function of $(r(t), R(t))$. Substituting these known functions of $(r(t), R(t))$ into the right-hand sides of (3.22), (3.23), or (3.24), (3.25) produces an autonomous system of two ordinary differential equations in the two unknowns $(r, R)$, the shock position $r$ and the cosmological scale factor $R$ of the FRW metric. These quantities then determine the FRW density $\rho(t)=\rho(\bar{r}(t))$, and the FRW pressure $p(t)=p(\bar{r}(t))$; cf. the note following (2.18).

Assume, then, that we have a smooth solution of (3.22), (3.23), or (3.24), (3.25). Reversing the steps (3.16) to (3.25) implies that (3.14) and (3.15) hold with $p=p_{+}$or $p=p_{-}$, respectively. The shock surface equation $\rho=\frac{3}{4 \pi} \frac{M(\bar{r})}{r^{3}}$ together with (3.20) then imply (2.33), so the solution $R(t), \rho(t), p(t)$ must satisfy the FRW equations (2.9), (2.10). Conservation then follows from (2.30) and Theorem 3. We have proved 
Theorem 7. Let a fixed OT solution satisfying (2.20) be given. Then any FRW metric that matches this OT metric Lipschitz continuously across the shock surface (2.18) such that (2.21), (2.22) and (2.36) hold, and such that the Rankine-Hugoniot jump conditions

$$
\left[T^{i j}\right] n_{i}=0
$$

also hold across the shock, must satisfy the ordinary differential equations (3.22), (3.23) or (3.24), (3.25). Conversely, any smooth solution of (3.22), (3.23) or (3.24), (3.25) satisfying (2.21), (2.22) and (2.36), will determine a solution of FRW type if

$$
\rho=\frac{3}{4 \pi} \frac{M}{\bar{r}^{3}},
$$

and $p$ is given by (3.9) or (3.10), respectively. This solution matches the OT metric Lipschitz continuously across the shock surface (2.18) (when the coordinate identification (2.23) is made), and the Rankine-Hugoniot jump conditions hold across the shock.

In the above problem we assumed as given the OT equation of state and solution, and we then determined the FRW pressure, and ordinary differential equations for shock solution. One can also consider the "inverse" problem of assigning the FRW equation of state and solution, and of trying to determine the OT pressure and corresponding ordinary differential equations for the shock solution. For the pressure, one can solve (3.9) for $\bar{p}$. An easy calculation gives

$$
\bar{p}=\frac{\theta \bar{\gamma} \rho-\bar{\rho}}{1-\theta \bar{\gamma}},
$$

where

$$
\bar{\gamma}=\frac{\bar{\rho}+p}{\rho+p} .
$$

Note the remarkable symmetry between (3.26) and (3.9). However, this symmetry does not carry over to the corresponding shock equations. Indeed, when the FRW variables are known functions of $t$, we need to replace $t$ with a known function of $\bar{r}$ and the unknown OT variables in order to derive a closed system of ordinary differential equations for $\bar{\rho}$ and $M$ as functions of $\bar{r}$. For this, one must go to the shock surface equation $M=\frac{4 \pi}{3} \rho \bar{r}^{3}$, and invert (the known) $\rho(t)$ in order to express $t$ as a known function of the two variables $M$ and $\bar{r}$. Moreover, in this case the conservation equation and the OT equations depend explicitly on $\bar{r}$ as well, and so fixing the FRW metric and solving for the OT metric leads to a considerably more complicated nonautonomous system of ordinary differential equations. The reason our approach is simpler and leads to an autonomous system of ordinary differential equations is because we can get $\bar{r}$ directly as a function of $r$ and $R$ from the identification $\bar{r}=R r$, and we can solve for $\rho$ as a known function of $\bar{r}$ from 
the shock-surface equation. Thus the conservation equation, as well as the FRW equations, is autonomous. This justifies the approach we have taken.

We now obtain the (invariant) shock speed relative to an observer fixed with the FRW fluid element. We recall that the "speed" of a shock is a coordinate-dependent quantity that can be interpreted in a special relativistic sense at a point $P$ in coordinate systems for which $g_{i j}(P)=\operatorname{diag}(-1,1,1,1)$. (We call such coordinate frames "locally Minkowskian" to distinguish these from "locally Lorentzian" frames in which $g_{i j, k}(P)=0$ as well. Since we are dealing only with velocities and not accelerations, we do not need to invoke the additional condition $g_{i j, k}(P)=0$ for a local Lorentzian coordinate frame in order to recover a special relativistic interpretation for velocities.) Moreover, since we are dealing only with radial motion, it suffices to work with coordinate systems that are locally Minkowskian in the $(t, r)$-variables alone. In such coordinate frames, a "speed" at $P$ transforms according to the special relativistic velocity transformation law when a Lorentz transformation is performed. We now determine the shock speed at a point $P$ on the shock in a locally Minkowskian frame that is co-moving with the FRW metric. To this end, let $(t, r)$-coordinates correspond to the FRW metric (2.4). Let $(t, \tilde{r})$ coordinates correspond to a locally Minkowskian system obtained from $(t, r)$ by a transformation of the form $r=\varphi(\tilde{r})$, so that, in $(t, r)$-coordinates,

$$
d s^{2}=-d t^{2}+\frac{R(t)^{2}}{1-k r^{2}}\left(\varphi^{\prime}\right)^{2} d \tilde{r}^{2} .
$$

Choose $\varphi$ so that $\frac{R(t)^{2}}{1-k r^{2}}\left(\varphi^{\prime}\right)^{2}=1$ at the point $P$; i.e., at $P=P(t, r)$, set $\varphi^{\prime}(r)=\frac{\sqrt{1-k r^{2}}}{R(t)}$. Thus, in the $(t, \tilde{r})$-coordinates,

$$
d s^{2}=-d t^{2}+d \tilde{r}^{2}
$$

at the point $P$, and so the $(t, \tilde{r})$-coordinates represent the class of locally Minkowskian coordinate frames that are fixed relative to the fluid particles of the FRW metric at the point $P$. (That is, any two members of this class of coordinate frames differ by higher-order terms that do not affect the calculation of radial velocities at $P$.) Therefore, the speed $d \tilde{r} / d t$ of a particle in $(t, \tilde{r})$-coordinates gives the value of the speed of the particle relative to the FRW fluid in the special relativistic sense. Since

$$
\frac{d r}{d t}=\frac{d r}{d \tilde{r}} \frac{d \tilde{r}}{d t}=\varphi^{\prime} \frac{d \tilde{r}}{d t}=\frac{\sqrt{1-k r^{2}}}{R} \frac{d \tilde{r}}{d t},
$$

we conclude that if the speed of a particle in $(t, r)$-coordinates is $d r / d t$, then its geometric speed relative to observers fixed with the FRW fluid (and hence also fixed relative to the radial coordinate $r$ of the FRW metric because the fluid is co-moving) is equal to $\frac{R}{\sqrt{1-k r^{2}}} \frac{d r}{d t}$.

Thus, let

$$
s=\frac{d \tilde{r}}{d t}=\frac{R}{\sqrt{1-k r^{2}}} \frac{d r}{d t}
$$


be the shock speed relative to the FRW fluid as measured in the local Minkowski frame fixed relative to the FRW fluid element. Then by (3.23) the speed $s_{+}^{2}$ for pressure $p_{+}$is given by

$$
s_{+}^{2}=\frac{1-\theta}{(1-\Theta)^{2}} \text {. }
$$

Thus the condition that the shock speed be less than the speed of light is

$$
\frac{1-\theta}{(1-\Theta)^{2}}<1
$$

Substituting $\Theta=\gamma \theta$ into (3.31) yields

$$
\theta>\frac{2 \gamma-1}{\gamma^{2}}=1-\left(\frac{1-z}{1+\bar{\mu} z}\right)^{2} \equiv \theta_{-} .
$$

Substituting

$$
\theta=\frac{A}{1-k r^{2}}, \quad \gamma=\frac{\rho+\bar{p}}{\bar{\rho}+\bar{p}}
$$

into (3.32) and using the identity

$$
\frac{2 \gamma-1}{\gamma^{2}}=1-\left(\frac{\rho-\bar{\rho}}{\rho+\bar{p}}\right)^{2}
$$

yields the expression

$$
A>\left(1-k r^{2}\right)\left(\frac{\rho-\bar{\rho}}{\rho+\bar{p}}\right)^{2}+k r^{2}
$$

This proves

Theorem 8. Both (3.32) and (3.33) are equivalent to the condition that the shock speed $s_{+}$be less than the speed of light on solutions of (3.22), (3.23) when $p \equiv p_{+}$.

When we take $p \equiv p_{-}$, we obtain $s_{-}^{2}$ by substituting $\theta$ for $\Theta$ in (3.30), namely,

$$
s_{-}^{2}=\frac{1}{1-\theta} .
$$

Since $\frac{1}{1-\theta}>1$ when $\theta<1$, we conclude that (3.34) rules out shocks with $p=p_{-}$as physical when $\theta<1$. (This rules out $p_{-}$as physically possible for the FRW pressure when $A>0$. In Section 5 we show by another argument that even if $p=p_{+}$, the sound speed is not positive when $\bar{\rho}>\rho$; this rules out implosions as physically meaningful when the FRW metric is inside the OT metric. Thus the only physically interesting case left when $A>0$ is when $p=p_{+}$and $z \equiv \bar{\rho} / \rho<1$, the case of an explosion when the FRW metric is on the inside.)

The following lemma gives a simple expression for the shock speed as measured in the OT barred coordinate frame in the case $p=p_{+}$: 
Lemma 1. Consider any solution of the shock ordinary differential equations (3.22), (3.23) when the pressure $p_{+}$is given by (3.9). Then the speed $d \bar{r} / d \bar{t}$ of the shock surface $r(t)$ as measured in the OT barred coordinate frame is given by

$$
\left(\frac{d \bar{r}}{d \bar{t}}\right)^{2}=\left(\frac{\gamma}{\gamma-1}\right)^{2}(1-\theta) A B
$$

Proof. We use the identities (4.51), (4.33), (4.42), (4.54), (8.1), respectively, derived in [6]:

$$
\begin{aligned}
C & =R^{2} A, \\
E & =-R \dot{R} \bar{r}, \\
\psi^{2} & =\frac{1}{B\left(R^{2}-k \bar{r}^{2}\right) C}, \\
\dot{R}^{2} r^{2} & =-A+1-k r^{2}, \\
d \bar{t} & =(\psi C-\psi E \dot{R} r) d t-\psi E R d r .
\end{aligned}
$$

Now note that (3.36) and (3.37) imply that

$$
-\frac{E}{C}=\frac{\dot{R} r}{A},
$$

and that (3.36) and (3.38) imply that

$$
\psi^{2} C^{2}=\frac{A}{B\left(1-k r^{2}\right)} .
$$

By (3.40),

$$
\frac{\partial \bar{t}}{\partial t}=\psi C-\psi E \dot{R} r=\psi C\left(1-\frac{E}{C} \dot{R} r\right),
$$

so using (3.41), (3.42) and (3.39), we obtain

$$
\begin{aligned}
\left(\frac{\partial \bar{t}}{\partial t}\right)^{2} & =\psi^{2} C^{2}\left(1-\frac{E}{C} \dot{R} r\right)^{2}=\frac{A}{B\left(1-k r^{2}\right)}\left(1+\frac{\dot{R} r}{A}\right)^{2} \\
& =\frac{A}{B\left(1-k r^{2}\right)}\left(1+\frac{-A+1-k r^{2}}{A}\right) \\
& =\frac{1-k r^{2}}{A B}=\frac{1}{\theta B} .
\end{aligned}
$$

Next, from (3.40), we get

$$
\left(\frac{\partial \bar{t}}{\partial r}\right)^{2}=\psi^{2} E^{2} R^{2}
$$

so using (3.37)-(3.39), we have 


$$
\begin{aligned}
\left(\frac{\partial \bar{t}}{\partial r}\right)^{2} & =\frac{R^{4} \dot{R} \bar{r}^{2}}{\left(1-k r^{2}\right) A B}=\frac{R^{2}\left(-A+1-k r^{2}\right)}{\left(1-k r^{2}\right) A B} \\
& =\frac{R^{2}}{B}\left(\frac{-1}{1-k r^{2}}+\frac{1}{A}\right)=\frac{R^{2}}{A B}(1-\theta)
\end{aligned}
$$

Now

$$
\bar{r}(\bar{t})=\bar{r}(\bar{t}(t, r))
$$

so that

$$
\frac{d \bar{r}}{d t}=\frac{d \bar{r}}{d \bar{t}}\left(\frac{\partial \bar{t}}{\partial t}+\frac{\partial \bar{t}}{\partial r} \dot{r}\right)
$$

and using (3.45) and (3.46) we find

$$
\frac{d \bar{r}}{d t}=\frac{d \bar{r}}{d \bar{t}} \frac{1}{\sqrt{\theta B}} \frac{\theta(\gamma-1)}{\gamma \theta-1},
$$

so

$$
\frac{d \bar{r}}{d \bar{t}}=\frac{d \bar{r}}{d t} \frac{\sqrt{\theta B}(\gamma \theta-1)}{\theta(\gamma-1)} .
$$

But adding (3.22) and (3.23) gives

$$
\dot{\bar{r}}=\sqrt{1-k r^{2}} \sqrt{1-\theta} \frac{\gamma \theta}{\gamma \theta-1} .
$$

Therefore,

$$
\frac{d \bar{r}}{d \bar{t}}=\sqrt{1-k r^{2}} \sqrt{1-\theta} \frac{\gamma \theta}{\gamma \theta-1} \frac{\sqrt{\theta B}(\gamma \theta-1)}{\theta(\gamma-1)}=\frac{\gamma}{\gamma-1} \sqrt{A B} \sqrt{1-\theta},
$$

which proves the lemma.

We now briefly discuss the significance of (3.31)-(3.33). Note that $A$ and $\gamma$ are determined by the OT solution and $\bar{r}$ alone. For the ordinary differential equations (3.22), (3.23), we are free to choose two initial conditions $r$ and $R$. Moreover, the OT solution is determined by the choice of initial conditions $M$ and $\bar{\rho}$ at given $\bar{r}$ for arbitrary equation of state $\bar{p}=\bar{p}(\bar{\rho})$. Therefore we can determine local shock wave solutions by arbitrarily assigning the OT equation of state, as well as $\bar{r}, \bar{\rho}, M$ and one of $r$ or $R$ (because $R r=\bar{r}$ ), thus allowing four initial conditions in all. Note that as $\gamma \rightarrow 1$ (which is equivalent to $\rho \rightarrow \bar{\rho}$, the weak shock limit) in (3.32),

$$
\frac{2 \gamma-1}{\gamma^{2}} \rightarrow 1
$$

so $\theta \rightarrow 1$ and the shock speed tends to zero.

We now consider the problem of determining when the Lax shock conditions hold for the shocks determined by (3.22), (3.23). To this end, we first 
find the OT fluid velocity as measured in the local Minkowski coordinate frame fixed with the FRW fluid. Using the identities

$$
\frac{d r}{d t}=\frac{\sqrt{1-k r^{2}}}{R} \frac{d \tilde{r}}{d t}, \quad \frac{d \bar{r}}{d t}=\dot{r} R+r \dot{R}=0,
$$

for the speeds of the OT fluid, we obtain

$$
\tilde{u}=\frac{R \dot{r}}{\sqrt{1-k r^{2}}}=-\frac{r \dot{R}}{\sqrt{1-k r^{2}}},
$$

where $\tilde{u}$ denotes the velocity $d \tilde{r} / d t$ of the OT fluid as measured in a locally Minkowskian coordinate frame fixed relative to the FRW fluid. Thus by (2.35),

$$
\tilde{u}=-\sqrt{1-\theta}
$$

When $\theta<1$, (3.51) implies

$$
|\tilde{u}|<1 \text {. }
$$

We now find expressions for the Lax shock condition in the case when the shock is an outgoing 2 -shock $(\rho>\bar{\rho}$, the only physically interesting case remaining when $A>0$ ), and the FRW metric is inside the OT metric. The Lax shock conditions express the requirement that the characteristics in the family of the shock impinge on the shock, and all other characteristics cross the shock. For a 2-shock the Lax shock conditions are (cf. [4])

$$
\begin{array}{lr}
\sqrt{\sigma}>s & (\text { FRW }- \text { Lax }), \\
s>\tilde{\lambda}_{2}^{\text {OT }} & (\text { OT }- \text { Lax }),
\end{array}
$$

where

$$
\sigma \equiv \frac{d p}{d \rho}=\frac{\dot{p}}{\dot{\rho}}=\frac{p^{\prime}}{\rho^{\prime}}
$$

denotes the FRW sound speed, and $\tilde{\lambda}_{2}^{O T}$ denotes the characteristic speed of the outgoing OT sound wave as measured in the $(t, \tilde{r})$ coordinate system. Here we let the dot denote $d / d t$ and the prime denote $d / d \bar{r}$.

To simplify (3.54), we recall that the OT characteristic speed $\tilde{\lambda}_{2}^{O T}$ is obtained by using the relativistic addition of velocities formula to add the velocities $\tilde{u}$ to $\sqrt{\bar{\sigma}}$, where

$$
\bar{\sigma} \equiv \frac{d \bar{p}}{d \bar{\rho}}
$$

i.e.,

$$
\tilde{\lambda}_{2}^{O T}=\frac{\tilde{u}+\sqrt{\bar{\sigma}}}{1+\tilde{u} \sqrt{\bar{\sigma}}}
$$


Thus for outgoing shocks with $p_{+}>0$ (which implies that $\Theta-1>0$ by (3.12)), (3.54) is equivalent to

$$
\frac{\sqrt{1-\theta}}{(\Theta-1)}>\tilde{\lambda}_{2}^{O T}=\frac{\tilde{u}+\sqrt{\bar{\sigma}}}{1+\tilde{u} \sqrt{\bar{\sigma}}} .
$$

Using (3.51) and simplifying, we obtain (cf. (3.12))

$$
(1-\sqrt{\bar{\sigma}} \sqrt{1-\bar{\sigma}}) \sqrt{1-\bar{\sigma}}>(-\sqrt{1-\theta}+\sqrt{\bar{\sigma}})(\Theta-1),
$$

which, after simplying and squaring, leads to

$$
\theta<1-\left(\frac{\gamma-1}{\gamma}\right)^{2} \bar{\sigma} .
$$

But

$$
\frac{\gamma-1}{\gamma}=\frac{\frac{\frac{1}{z}+\bar{\mu}}{\frac{1+\bar{\mu}}{1+\bar{\mu}}-1}}{\frac{\frac{1}{z}+\bar{\mu}}{1+\bar{\mu}}}=\frac{1-z}{1+\bar{\mu} z},
$$

and

$$
\frac{1-z}{1+\bar{\mu} z}<1
$$

for $0 \leqq \bar{\mu}<1$ and $0<z<1$, and so the OT-Lax condition (3.54) reduces to

$$
\theta<1-\left(\frac{1-z}{1+\bar{\mu} z}\right)^{2} \bar{\sigma} \equiv \theta_{+}(z, \bar{\mu}, \bar{\sigma}) .
$$

Now if we set $\mu=p_{+} / \rho$, then by (3.9)

$$
\mu=\frac{\gamma \theta z-1}{1-\gamma \theta} .
$$

By using (3.64) to solve for $\theta$ in the inequality $\mu<1$, it is straightforward to verify that the (physically interesting) condition $0<\mu<1$ is equivalent to (cf. (3.11))

$$
\theta>\frac{2 z(1+\bar{\mu})}{(1+z)(1+\bar{\mu} z)} \equiv \theta_{2}(z, \bar{\mu}) \geqq \theta_{1}(z, \bar{\mu})
$$

We use

Lemma 2. The following inequalities hold for $z>0$ :

$$
\begin{gathered}
\theta_{1}<\theta_{2}<\theta_{-}<\theta_{+}, \\
\theta_{-}<4 z .
\end{gathered}
$$


Proof of Lemma 2. By (3.32),

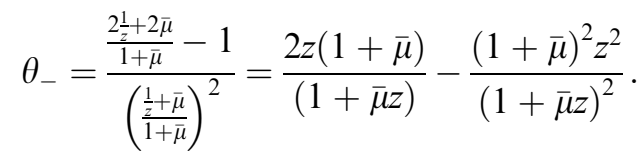

By neglecting the negative term in (3.68) and estimating $|\bar{\mu}|<1$ we see immediately that

$$
\theta_{-}<4 z
$$

Moreover, from (3.68) we can also estimate

$$
\begin{aligned}
\theta_{-} & =\frac{2 z(1+\bar{\mu})(1+z)}{(1+z)(1+\bar{\mu} z)}-\frac{(1+\bar{\mu})^{2} z^{2}}{(1+\bar{\mu} z)^{2}} \\
& =\theta_{2}+\frac{2 z^{2}(1+\bar{\mu})}{(1+z)(1+\bar{\mu} z)}-\frac{z^{2}(1+\bar{\mu})^{2}}{(1+\bar{\mu} z)^{2}} \\
& >\theta_{2},
\end{aligned}
$$

because

$$
\frac{2 z^{2}(1+\bar{\mu})}{(1+z)(1+\bar{\mu} z)}-\frac{z^{2}(1+\bar{\mu})^{2}}{(1+\bar{\mu} z)^{2}}=\frac{z^{2}(1+\bar{\mu})(1-z)(1-\bar{\mu})}{(1+\bar{\mu} z)^{2}(1+z)}>0 .
$$

The inequality $\theta_{1}<\theta_{2}$ follows directly from (3.11). Finally,

$$
\theta_{+}-\theta_{-}=\left(\frac{\gamma-1}{\gamma}\right)^{2}(1-\bar{\sigma})>0
$$

in light of the identity

$$
\frac{\gamma-1}{\gamma}=\frac{1-z}{1+\bar{\mu} z}
$$

This proves the lemma.

We can now state and prove

Theorem 9. Assume that an OT solution $\bar{\rho}>0, \bar{p}(\bar{r})>0$ and $M(\bar{r})>0$ of (2.14), (2.15) is defined and smooth for all $\bar{r}$ in the interval

$$
\bar{r}_{-}<\bar{r}<\bar{r}_{+} \leqq \infty .
$$

Assume also that the following additional conditions hold throughout the interval $\left(\bar{r}_{-}, \bar{r}_{+}\right)$:

$$
\begin{aligned}
& 0<\bar{\mu}=\frac{\bar{p}(\bar{r})}{\bar{\rho}(\bar{r})}<1, \\
& 0<\bar{\sigma}=\frac{\bar{p}^{\prime}(\bar{r})}{\bar{\rho}^{\prime}(\bar{r})}<1,
\end{aligned}
$$




$$
\rho=\frac{3}{4 \pi} \frac{M(\bar{r})}{\bar{r}^{3}}>\bar{\rho} .
$$

Then the solution $(r(t), R(t))$ of the shock equations $(3.22)_{+}$and (3.23) _ starting from initial data $\left(r_{0}, R_{0}\right)$ satisfying

$$
\bar{r}_{-}<\bar{r}_{0}=r_{0} R_{0}<\bar{r}_{+}
$$

exists, and determines an outgoing shock wave that satisfies

$$
\begin{gathered}
0<s<1, \\
p>\bar{p}, \\
\rho>\bar{\rho}, \\
0<\mu=\frac{p}{\rho}<1,
\end{gathered}
$$

together with the OT-Lax condition (3.54) throughout the maximal sub-interval of $\left(\bar{r}_{-}, \bar{r}_{+}\right)$containing $\bar{r}_{0}$ on which

$$
\theta_{-}<\theta<\theta_{+}
$$

where

$$
\begin{aligned}
& \theta_{-} \equiv \theta_{-}(z, \bar{\mu})=\frac{2 \gamma-1}{\gamma^{2}}=1-\left(\frac{1-z}{1+\bar{\mu} z}\right)^{2}, \\
& \theta_{+} \equiv \theta_{+}(z, \bar{\mu}, \bar{\sigma}) \equiv 1-\left(\frac{1-z}{1+\bar{\mu}}\right)^{2} \bar{\sigma}
\end{aligned}
$$

Corollary 1. Condition (3.79) is implied by the simpler, less sharp condition

$$
4 z<\theta<1-\frac{\bar{\sigma}}{(1+\bar{\mu})^{2}} \text {. }
$$

Proof of Theorem 9. The only obstruction to existence for (3.22), (3.23) is $\Theta=\gamma \theta=1$, the latter occuring at $\theta=\theta_{1}$; cf. equation (3.11). Since $\theta_{1}<\theta_{-}$, existence is clear. Moreover, (3.75), (3.78) and the OT-Lax conditions hold in light of (3.32), (3.65) and (3.63). The verification of (3.82) follows directly from (3.66) and (3.81). That (3.76) and (3.77) hold follows from Theorem 5. This proves the theorem and corollary.

In the next section we derive the following formula for the sound speed $\sigma$ :

$$
(1-\theta \gamma)^{2} \sigma=\frac{1}{6} \frac{\theta(1-A)}{A(1+\bar{\mu})}\left\{\alpha(z, \bar{\mu})+\beta(z, \bar{\mu}) \frac{1-\theta}{\bar{\sigma}}\right\}+\frac{2}{3} \frac{\theta}{A}+\theta-\frac{5}{3},
$$

where 


$$
\begin{aligned}
& \alpha(z, \bar{\mu}) \equiv \frac{3-7 z+5 \bar{\mu} z-9 \bar{\mu} z^{2}}{z}, \\
& \beta(z, \bar{\mu}) \equiv \frac{(1+3 \bar{\mu} z)(1+\bar{\mu} z)^{2}}{z(1-z)} .
\end{aligned}
$$

Using this, it is not difficult to show that the FRW-Lax shock condition (3.53) is equivalent to

$$
\frac{1}{6} \frac{\theta(1-A)}{A(1+\bar{\mu})}\left\{\alpha(z, \bar{\mu})+\beta(z, \bar{\mu}) \frac{(1-\theta)}{\bar{\sigma}}\right\}+\frac{2}{3} \frac{\theta}{A}+2 \theta>\frac{8}{3} .
$$

Note now that $\theta_{-} \rightarrow 0$ and $\theta_{+} \rightarrow 1-\frac{\bar{\sigma}}{(1+\bar{\mu})^{2}}>1-\bar{\sigma}$ as $z \rightarrow 0$. Moreover, note that $\alpha, \beta$ and $\gamma$ tend to $+\infty$ like $\frac{1}{z}$ as $z \rightarrow 0$. Using this it is not difficult to verify the following theorem which states (roughly) that for outgoing shocks, the Lax characteristic conditions (3.53) and (3.54) hold, and the FRW sound speed is positive and less than the speed of light, if the shock is sufficiently strong. This demonstrates that system (3.22), (3.23) generates a large set of physically meaningful shock-wave solutions of the Einstein equations that model explosions.

Theorem 10. Assume that (2.20)-(2.22) hold and let $p=p_{+}$. Fix the dimensionless constants $\bar{\mu}, \bar{\sigma}, A$, and $\theta$ (assume that $0<A<\theta<1$ if $k>0$ or $0<\theta \leqq A$ if $k \leqq 0)$ such that (3.79) holds. If $z=\bar{\rho} / \rho$ is sufficiently small, then $0<\sigma<1, p>\bar{p}$, and the FRW-Lax shock conditions (3.53) and (3.54) both hold.

Since the FRW equation of state is determined by the OT equation of state through the ordinary differential equations (3.22), (3.23), there remains the question of how the FRW equation of state is restricted by these equations. Assume an outgoing shock with $\rho>\bar{\rho}$. We now show that given any values of $\bar{\sigma}_{0}, \bar{\mu}_{0}, \sigma_{0}, \mu_{0}$ and $\rho_{0}>0$ satisfying

$$
0<\bar{\sigma}_{0}, \bar{\mu}_{0}, \sigma_{0}, \mu_{0}<1 \text {, }
$$

there exists a range of values of $z_{0}$, near $z=0$, such that, when $\bar{\sigma}_{0}, \bar{\mu}_{0}, \sigma_{0}, \mu_{0}, \rho_{0}$ and $z_{0}$ are taken as initial values for (3.22), (3.23) and the outer OT solution, then the shock-wave solution so determined is a Lax shock wave which moves at less than the speed of light in some neighborhood of the initial point. This demonstrates (roughly) that, for sufficiently strong shocks, arbitrary equations of state can be approximated locally on each side of the shock in this theory. Note that $\sigma_{0}, \mu_{0}, \rho_{0}$ and $z_{0}$ supply the requisite number of initial conditions, two for the OT equations (2.14), (2.15) and two for the shock equations (3.22), (3.23). In the next theorem we show that even after prescribing $\bar{\mu}_{0}$ and $\bar{\sigma}_{0}$ for the OT equation of state at a point, we still have enough freedom in the initial conditions to freely assign $\sigma_{0}, \mu_{0}, \rho_{0}$, and $z_{0}$, at a point. To state the theorem, note first that for any values of $\bar{\sigma}_{0}, \bar{\mu}_{0}, \sigma_{0}$ and $\mu_{0}$, satisfying (3.87), we can use 


$$
\mu=\frac{\gamma \theta z-1}{1-\gamma \theta}
$$

to solve for $\theta$ and obtain

$$
\theta=\frac{\left(1+\bar{\mu}_{0}\right)\left(1+\mu_{0}\right)}{\left(1+\bar{\mu}_{0} z\right)\left(z+\mu_{0}\right)} z
$$

It is readily shown that when (3.87) holds, equation (3.89) defines $\theta$ as a continuous function of $z$ that takes on all values from 0 to 1 as $z$ increases from 0 to 1 .

Theorem 11. For any choice of $\bar{\sigma}_{0}, \bar{\mu}_{0}, \sigma_{0}, \mu_{0}$ satisfying (3.87), and any choice of $z_{0}, \theta_{0} \in(0,1)$ such that

$$
\theta_{0}=\frac{\left(1+\bar{\mu}_{0}\right)\left(1+\mu_{0}\right)}{\left(1+\bar{\mu}_{0} z\right)\left(z+\mu_{0}\right)} z_{0}
$$

and such that

$$
\theta_{0}<1-\bar{\sigma}
$$

there exists $A_{0} \in(0,1)$ such that (cf. (3.83))

$$
\sigma_{0}=\left(1-\gamma_{0} \theta_{0}\right)^{-2}\left\{\frac{1}{6} \frac{\theta_{0}}{A_{0}} \frac{\left(1-A_{0}\right)}{\left(1+\bar{\mu}_{0}\right)}\left(\alpha_{0}+\beta_{0} \frac{1-\theta_{0}}{\bar{\sigma}_{0}}\right)+\frac{2}{3} \frac{\theta_{0}}{A_{0}}+\theta_{0}-\frac{5}{3}\right\} .
$$

Before proving the theorem, we make a few remarks. First, we conclude from the theorem that the choice of values $A_{0}, \mu_{0}, \rho_{0}$ and $z_{0}$ (together with a given OT equation of state satisfying $\frac{d \bar{p}}{d \bar{\rho}}\left(\bar{\rho}_{0}\right) \equiv \bar{\sigma}_{0}$ and $\left.\frac{\bar{p}\left(\bar{\rho}_{0}\right)}{\bar{\rho}_{0}} \equiv \bar{\mu}_{0}\right)$ uniquely determines the initial conditions for the OT ordinary differential equations (2.9), (2.10) and the shock ordinary differential equations (3.22), (3.23). To see this, note that the shock surface condition $M=\frac{4 \pi}{3} \rho \bar{r}^{3}$ and the identity $A=1-2 \mathscr{G} M / \bar{r}$ determine $M_{0}$ and $\bar{r}_{0}$ from $\rho_{0}$ and $A_{0}$, and $\bar{\rho}_{0}$ is determined from $\bar{\rho}_{0}=z_{0} \rho_{0}$. Thus, assuming any OT equation of state that satisfies $\bar{p}_{0}=\bar{\mu}_{0} \bar{\rho}_{0}$ and $\frac{d \bar{p}}{d \bar{\rho}}\left(\bar{\rho}_{0}\right)=\bar{\sigma}_{0}$, we obtain a (local) OT solution from (2.14), (2.15). We obtain $r_{0}$ and $R_{0}$ from the identity $\theta=\frac{A}{1-k r^{2}}$, thus supplying the remaining initial conditions required for (3.22), (3.23). Furthermore, if we restrict the choice of $z_{0}$ so that (3.89) implies (cf. (3.32), (3.80) and (3.81))

$$
\theta_{-}<\theta_{0}<\theta_{+}, \quad \theta_{0}>\frac{2 \gamma_{0}-1}{\gamma_{0}^{2}}
$$

then the resulting solution is (locally) a Lax shock, and the shock speed is (locally) less than the speed of light. Moreover, it is straightforward to verify that as $z_{0} \rightarrow 0$ (strong shocks), (3.90) implies that $\theta_{0}$ asymptotically looks like

$$
\theta_{0} \approx \frac{\left(1+\bar{\mu}_{0}\right)\left(1+\mu_{0}\right)}{\mu_{0}} z
$$


while $\theta_{-}$and $\frac{2 \gamma_{0}-1}{v^{2}}$ asymptotically look like $2\left(1+\bar{\mu}_{0}\right) z_{0}$, and so $\theta_{0}<\theta_{+}$, and both $\theta_{-}<\theta_{0}$, and $\frac{2 \gamma_{0}-1}{\gamma^{2}}<\theta_{0}<\theta_{+}$, for sufficiently small $z_{0}$. Thus we have shown that the equation of state can be freely assigned at a point for sufficiently strong shocks.

Note too that $A<\theta$ for $k>0, A=\theta$ for $k=0$, and $A>\theta$ for $k>0$, and so the relative sizes of $A_{0}$ and $\theta_{0}$ determine $k$ uniquely. Note also that in the above, we could just as well have initially assigned $\bar{r}_{0}$ instead of $\rho_{0}$, etc., with the same conclusion.

Proof of Theorem 11. We use

Lemma 3. Assume that $\theta<1-\bar{\sigma}$. Then

$$
\alpha(z, \mu)+\beta(z, \mu) \frac{1-\theta}{\bar{\sigma}} \geqq \frac{3}{7 z(1-z)} .
$$

We can use this lemma to demonstrate the theorem as follows: By using (3.93) and (3.83) we can estimate

$$
\begin{aligned}
(1-\theta \gamma)^{2} \sigma & \geqq \frac{1}{6} \frac{\theta(1-A)}{A(1+\bar{\mu})} \frac{3}{7 z(1-z)}+\frac{2}{3} \frac{\theta}{A}+\theta-\frac{5}{3} \\
& =\theta\left\{\frac{(1-A)}{21(1+\bar{\mu}) A^{2} z(1-z)}+\frac{2}{3 A}+1\right\}-\frac{5}{3}
\end{aligned}
$$

But since $\theta<1$, we can see directly from the right-hand side of the formula (3.83) that $\sigma<0$ when $A=1$. Moreover, by (3.94) we see directly that $\sigma \rightarrow+\infty$ as $A \rightarrow 0$. Thus by continuity we know that for every choice of $\sigma_{0} \in(0,1)$, there exists $A_{0} \in(0,1)$ such that (3.92) holds. Thus the lemma implies the theorem.

Proof of Lemma 3. Since we assume $\theta<1-\bar{\sigma}$, it follows that

$$
\frac{1-\theta}{\bar{\sigma}} \geqq 1
$$

and thus (3.84) and (3.85) imply that to verify (3.93), it suffices to show that

$$
f(z, \bar{\mu}) \geqq \frac{3}{7},
$$

where

$$
f(z, \bar{\mu})=\left\{\left(3-7 z+5 \bar{\mu} z-9 \bar{\mu} z^{2}\right)(1-z)\right\}_{\mathrm{I}}+\left\{(1+3 \bar{\mu} z)(1+\bar{\mu} z)^{2}\right\}_{\mathrm{II}} .
$$

Simplifying gives

$$
\begin{aligned}
& \{\}_{\mathrm{I}}=3+(-10+5 \bar{\mu}) z+(7-14 \bar{\mu}) z^{2}+9 \bar{\mu} z^{3}, \\
& \{\}_{\mathrm{II}}=1+5 \bar{\mu} z+7 \bar{\mu}^{2} z^{2}+3 \bar{\mu}^{3} z^{3} .
\end{aligned}
$$


Thus

$$
f(z, \bar{\mu})=\{\}_{\mathrm{I}}+\{\}_{\mathrm{II}}=4+10(\bar{\mu}-1) z+7(\bar{\mu}-1)^{2} z^{2}+9 \bar{\mu} z^{3} .
$$

But for fixed $\bar{\mu}$,

$$
g(z) \equiv 4+10(\bar{\mu}-1) z+7(\bar{\mu}-1)^{2} z^{2}
$$

takes a minimum value at

$$
z=z_{*} \equiv \frac{5}{7(1-\bar{\mu})}
$$

and

$$
g\left(z_{*}\right)=\frac{3}{7}
$$

independent of $\bar{\mu}$. Thus

$$
f(z, \bar{\mu}) \geqq \frac{3}{5}+9 \bar{\mu} z^{3} \geqq \frac{3}{5}
$$

gives (3.96), and this estimate is sharp as $\bar{\mu} \rightarrow 0$ or $z \rightarrow 0$.

In the next section we shall derive the formula (3.83) for the FRW sound speed.

\section{The FRW sound speed} (3.83):

In this section we prove the following theorem which was anticipated in

Theorem 12. The $F R W$ sound speed $\sigma \equiv \frac{d p}{d \rho}$, as determined by the shock equations (3.20), (3.21) is given by (3.83).

In order to calculate the FRW sound speed $\sigma \equiv \dot{p} / \dot{\rho}$ for any solution of (3.22), (3.23), recall that the pressure $p_{+}$is given by

$$
p_{+}=\frac{\Theta \bar{\rho}-\rho}{1-\Theta}
$$

where

$$
\Theta=\theta \gamma, \quad \gamma=\frac{\rho+\bar{p}}{\bar{\rho}+\bar{p}}, \quad \theta=\frac{A}{1-k r^{2}} .
$$

(The formulas for $p_{-}$could be obtained from the formulas for $p_{+}$by replacing $\Theta$ by $\theta$, or equivalently by replacing $\gamma$ by 1 in the formulas to follow.)

Let the OT equation of state $\bar{p}=\bar{p}(\bar{\rho})$ be given satisfying (2.20), let the OT sound speed be given by $\sqrt{\bar{\sigma}}$, where

$$
\frac{d \bar{p}}{d \bar{\rho}}=\bar{\sigma},
$$

and let $\bar{\mu}$ be defined by 


$$
\bar{p}=\bar{\mu} \bar{\rho} .
$$

We now calculate $\sigma=p_{+}^{\prime} / \rho^{\prime}$, where "prime" denotes differentiation with respect to $\bar{r}$, in terms of the following three dimensionless quantities that evolve according to (3.22), (3.23):

$$
\theta=\frac{A}{1-k r^{2}}, \quad A=1-\frac{2 \mathscr{G} M}{\bar{r}}, \quad z=\frac{\bar{\rho}}{\rho} .
$$

Differentiating (4.1) with respect to $\bar{r}$, and denoting $p_{+}$by $p$ (for notational convenience), we obtain

$$
p^{\prime}=p_{\theta} \theta^{\prime}+p_{\gamma} \gamma^{\prime}+p_{\bar{\rho}} \bar{\rho}^{\prime}+p_{\rho} \rho^{\prime} .
$$

Now from (4.1), we have

$$
p_{\theta}=\frac{(1-\theta \gamma)(\gamma \bar{\rho})-(\theta \gamma \bar{\rho}-\rho)(-\gamma)}{(1-\theta \gamma)^{2}} ;
$$

thus

$$
p_{\theta}=\frac{\gamma(\bar{\rho}-\rho)}{(1-\theta \gamma)^{2}}
$$

Similarly, we have

$$
\begin{aligned}
& p_{\gamma}=\frac{\theta(\bar{\rho}-\rho)}{(1-\theta \gamma)^{2}}, \\
& p_{\bar{\rho}}=\frac{\theta \gamma}{1-\theta \gamma}, \\
& p_{\rho}=\frac{-1}{1-\theta \gamma} .
\end{aligned}
$$

Furthermore,

$$
\theta^{\prime}=\frac{A^{\prime}}{1-k r^{2}}+\frac{2 k r A}{\left(1-k r^{2}\right)^{2}} \frac{d r}{d \bar{r}}
$$

Now

$$
A^{\prime}=-\frac{2 \mathscr{G} M^{\prime}}{\bar{r}}+\frac{2 \mathscr{G} M}{\bar{r}^{2}},
$$

which simplifies to

$$
A^{\prime}=8 \pi \mathscr{G} \bar{r}\left(\frac{1}{3} \rho-\bar{\rho}\right),
$$

and using (2.39) and (2.40), we have

$$
\frac{d r}{d \bar{r}}=\frac{d r}{d t} \frac{d t}{d \bar{r}}=\frac{\dot{r}}{\dot{\bar{r}}}=\frac{\dot{R} r(\bar{\rho}+p)}{R(\rho-\bar{\rho})} \frac{(\rho-\bar{\rho})}{\dot{R} r(\rho+p)},
$$


which simplifies to

$$
\frac{d r}{d \bar{r}}=\frac{\bar{\rho}+p}{R(\rho+p)}
$$

But by (4.1),

$$
p=\frac{\theta \gamma \bar{\rho}-\rho}{1-\theta \gamma}
$$

so that

$$
\frac{d r}{d \bar{r}}=\frac{1}{R} \frac{\bar{\rho}+\frac{\theta \gamma \bar{\rho}-\rho}{1-\theta \gamma}}{\rho+\frac{\theta \gamma \bar{\rho}-\rho}{1-\theta \gamma}}=\frac{1}{R} \frac{\bar{\rho}(1-\theta \gamma)+\theta \gamma \bar{\rho}-\rho}{\rho(1-\theta \gamma)+\theta \gamma \bar{\rho}-\rho}
$$

thus

$$
\frac{d r}{d \bar{r}}=\frac{1}{R \theta \gamma}
$$

Now from (4.9), using (4.11) and (4.15), we obtain

$$
\begin{aligned}
\theta^{\prime} & =\frac{8 \pi \mathscr{G} \bar{r}}{1-k r^{2}}\left(\frac{1}{3} \rho-\bar{\rho}\right)+\frac{\theta}{1-k r^{2}}(2 k r) \frac{1}{R \theta \gamma} \\
& =\frac{8 \pi \mathscr{G} \bar{r}}{1-k r^{2}}\left(\frac{1}{3} \rho-\bar{\rho}\right)+\frac{2 k r^{2}}{1-k r^{2}} \frac{1}{\bar{r} \gamma} \\
& =\frac{1}{\left(1-k r^{2}\right) \bar{r} \gamma}\left[8 \pi \mathscr{G}\left(\frac{1}{3}-z\right) \rho \bar{r}^{2} \gamma+2 k r^{2}\right]
\end{aligned}
$$

But

$$
\begin{gathered}
\rho \bar{r}^{2}=\frac{3}{8 \pi \mathscr{G}}(1-A), \\
1-k r^{2}=\frac{A}{\theta},
\end{gathered}
$$

so that

$$
k r^{2}=1-\frac{A}{\theta}
$$

Now using (4.17)-(4.19) in (4.16) gives

$$
\theta^{\prime}=\frac{1}{\bar{r} \gamma} \frac{\theta}{A}\left[(1-3 z)(1-A) \gamma+2\left(1-\frac{A}{\theta}\right)\right]
$$

Now 


$$
\begin{aligned}
\gamma^{\prime} & =\left(\frac{\rho+\bar{p}}{\bar{\rho}+\bar{p}}\right)^{\prime}=\frac{(\bar{\rho}+\bar{p})\left(\rho^{\prime}+\bar{p}^{\prime}\right)-(\rho+\bar{p})\left(\bar{\rho}^{\prime}+\bar{p}^{\prime}\right)}{(\bar{\rho}+\bar{p})^{2}} \\
& =\frac{(\bar{\rho}+\bar{p}) \rho^{\prime}+(\bar{\rho}+\bar{p}-\rho-\bar{p}) \bar{p}^{\prime}-(\rho+\bar{p}) \bar{\rho}^{\prime}}{(\bar{\rho}+\bar{p})^{2}}
\end{aligned}
$$

so that

$$
\gamma^{\prime}=\frac{1}{(\bar{\rho}+\bar{p})} \rho^{\prime}+\frac{(\bar{\rho}-\rho)}{(\bar{\rho}+\bar{p})^{2}} \bar{p}^{\prime}-\frac{\rho+\bar{p}}{(\bar{\rho}+\bar{p})^{2}} \bar{\rho}^{\prime}
$$

or

$$
\gamma^{\prime}=\frac{1}{(\bar{\rho}+\bar{p})} \rho^{\prime}-\frac{(\gamma-1)}{\bar{\rho}+\bar{p}} \bar{p}^{\prime}-\frac{\gamma}{\bar{\rho}+\bar{p}} \bar{\rho}^{\prime} .
$$

Thus we have

$$
\gamma^{\prime}=\frac{1}{\bar{\rho}+\bar{p}}\left\{\rho^{\prime}-(\gamma-1) \bar{p}^{\prime}-\gamma \bar{\rho}^{\prime}\right\} .
$$

Now using (4.2) and (4.3), we have

$$
\begin{aligned}
\vec{p}^{\prime} & =\bar{\sigma} \bar{\rho}^{\prime}=-4 \pi \mathscr{G} \bar{r}(\bar{\rho}+\bar{p})\left(\frac{1}{3} \rho+\bar{p}\right) A^{-1} \\
& =-4 \pi \mathscr{G} \bar{r}(1+\bar{\mu}) \bar{\rho}\left(\frac{1}{3}+\bar{\mu} z\right) \rho A^{-1}
\end{aligned}
$$

thus

$$
\vec{p}^{\prime}=-4 \pi \mathscr{G} z(1+\bar{\mu})\left(\frac{1}{3}+\bar{\mu} z\right) A^{-1} \rho^{2} \bar{r} .
$$

Since

$$
\rho^{\prime}=\frac{3(\bar{\rho}-\rho)}{\bar{r}},
$$

we have

$$
\rho^{\prime}=3 \frac{(z-1)}{\bar{r}} \rho .
$$

Thus

$$
\frac{\bar{p}^{\prime}}{\rho^{\prime}}=-\frac{4 \pi \mathscr{G}}{3} \frac{z}{z-1}(1+\bar{\mu})\left(\frac{1}{3}+\bar{\mu} z\right) A^{-1} \rho \bar{r}^{2}
$$

therefore

$$
\frac{\bar{p}^{\prime}}{\rho^{\prime}}=\frac{1}{6}\left(\frac{z}{1-z}\right)\left(\frac{1-A}{A}\right)(1+\bar{\mu})(1+3 \bar{\mu} z) .
$$

But

$$
\gamma-1=\frac{\frac{1}{z}+\bar{\mu}}{1+\bar{\mu}}-1=\frac{\frac{1}{z}-1}{1+\bar{\mu}}=\left(\frac{1-z}{1+\bar{\mu}}\right) \frac{1}{z},
$$

so that 


$$
\frac{z}{1-z}(1+\bar{\mu})=\frac{1}{\gamma-1},
$$

and thus

$$
\frac{\bar{p}^{\prime}}{\rho^{\prime}}=\frac{1}{6}\left(\frac{1-A}{A}\right)\left(\frac{1+3 \bar{\mu} z}{\gamma-1}\right)
$$

Hence

$$
\begin{aligned}
p_{\theta} \theta^{\prime} & =\frac{\gamma(\bar{\rho}-\rho)}{(1-\theta \gamma)^{2}} \frac{1}{\bar{r} \gamma} \frac{\theta}{A}\left[(1-3 z)(1-A) \gamma+2\left(1-\frac{A}{\theta}\right)\right] \\
& =\frac{(z-1)}{(1-\theta \gamma)^{2}} \frac{\theta}{A}\left[(1-3 z)(1-A) \gamma+2\left(1-\frac{A}{\theta}\right)\right] \frac{\rho}{\bar{r}} .
\end{aligned}
$$

Thus

$$
p_{\theta} \frac{\theta^{\prime}}{\rho^{\prime}}=\frac{z-1}{(1-\theta \gamma)^{2}} \frac{\theta}{A}\left[(1-3 z)(1-A) \gamma+2\left(1-\frac{A}{\theta}\right)\right] \frac{\rho}{\bar{r}} \frac{\bar{r}}{\rho} \frac{1}{3(z-1)} ;
$$

so

$$
p_{\theta} \frac{\theta^{\prime}}{\rho^{\prime}}=\frac{1}{3} \frac{1}{(1-\theta \gamma)^{2}} \frac{\theta}{A}\left[(1-3 z)(1-A) \gamma+2\left(1-\frac{A}{\theta}\right)\right] .
$$

Next, from (4.23), we have

$$
p_{\gamma} \gamma^{\prime}=\frac{\theta(\bar{\rho}-\rho)}{(1-\theta \gamma)^{2}} \frac{1}{\bar{\rho}+\bar{p}}\left[\rho^{\prime}-(\gamma-1) \overline{p^{\prime}}-\gamma \overline{\rho^{\prime}}\right],
$$

so that

$$
p_{\gamma} \frac{\gamma^{\prime}}{\rho^{\prime}}=\frac{\theta(\bar{\rho}-\rho)}{(1-\theta \gamma)^{2}} \frac{1}{\bar{\rho}+\bar{p}}\left[1-(\gamma-1) \frac{\overline{p^{\prime}}}{\rho^{\prime}}-\gamma \frac{\bar{\rho}^{\prime}}{\rho^{\prime}}\right]
$$

where

$$
\frac{\bar{p}^{\prime}}{\rho^{\prime}}=\bar{\sigma} \frac{\bar{\rho}^{\prime}}{\rho^{\prime}}=\frac{1}{6} \frac{(1-A)}{A} \frac{(1+3 \bar{\mu} z)}{\gamma-1} .
$$

But (4.30) further simplifies upon noting that

$$
\frac{\bar{\rho}-\rho}{\bar{\rho}+\bar{p}}=1-\gamma,
$$

so that

$$
p_{\gamma} \frac{\gamma^{\prime}}{\rho^{\prime}}=\frac{-\theta(\gamma-1)}{(1-\theta \gamma)^{2}}\left[1-(\gamma-1) \frac{\bar{p}^{\prime}}{\rho^{\prime}}-\gamma \frac{\bar{\rho}^{\prime}}{\rho^{\prime}}\right] .
$$

Now

$$
p_{\bar{\rho}} \bar{\rho}^{\prime}=\frac{\theta \gamma}{1-\theta \gamma} \bar{\rho}^{\prime}=\frac{\theta \gamma}{1-\theta \gamma} \frac{1}{\bar{\sigma}} \bar{p}^{\prime}, \quad p_{\rho} \rho^{\prime}=\frac{-1}{1-\theta \gamma} \rho^{\prime} ;
$$


thus

$$
\begin{aligned}
p_{\bar{\rho}} \frac{\bar{\rho}^{\prime}}{\rho^{\prime}} & =\frac{\theta \gamma}{1-\theta \gamma} \frac{1}{\bar{\sigma}} \frac{\bar{p}^{\prime}}{\rho^{\prime}}, \\
p_{\rho} & =\frac{-1}{1-\theta \gamma} .
\end{aligned}
$$

Therefore

$$
\sigma=\frac{p^{\prime}}{\rho^{\prime}}=p_{\theta} \frac{\theta^{\prime}}{\rho^{\prime}}+p_{\gamma} \frac{\gamma^{\prime}}{\rho^{\prime}}+p_{\bar{\rho}} \frac{\bar{\rho}^{\prime}}{\rho^{\prime}}+p_{\rho},
$$

where

$$
\begin{aligned}
p_{\theta} \frac{\theta^{\prime}}{\rho^{\prime}} & =\left\{\frac{1}{3} \frac{1}{(1-\theta \gamma)^{2}} \frac{\theta}{A}\left[(1-3 z)(1-A) \gamma+2\left(1-\frac{A}{\theta}\right)\right]_{a}\right\}_{\mathrm{I}}, \\
p_{\gamma} \frac{\gamma^{\prime}}{\rho^{\prime}} & =\left\{\frac{-\theta}{(1-\theta \gamma)^{2}}(\gamma-1)\left[1-(\gamma-1) \frac{\bar{p}^{\prime}}{\rho^{\prime}}-\gamma \frac{\bar{\rho}^{\prime}}{\rho^{\prime}}\right]_{b}\right\}_{\mathrm{II}}, \\
p_{\bar{\rho}} \frac{\bar{\rho}^{\prime}}{\rho^{\prime}} & =\left\{\frac{\theta \gamma}{1-\theta \gamma} \frac{\bar{\rho}^{\prime}}{\rho^{\prime}}\right\}_{\mathrm{III}}, \\
p_{\rho} & =\left\{\frac{-1}{1-\theta \gamma}\right\}_{\mathrm{IV}} .
\end{aligned}
$$

To simplify (4.34), we can distribute $\frac{-\theta}{(1-\theta \gamma)^{2}}(\gamma-1)$ over the three terms in []$_{b}$, and then add the first term to \{\}$_{\mathrm{IV}}$, and the third $\frac{\bar{\rho}^{\prime}}{\rho^{\prime}}$ term in []$_{b}$ to \{\}$_{\mathrm{III}}$ :

$$
p_{\gamma} \frac{\gamma^{\prime}}{\rho^{\prime}}=\left[\frac{-\theta(\gamma-1)}{(1-\theta \gamma)^{2}}\right]_{c}+\left[\frac{\theta(\gamma-1)}{(1-\theta \gamma)^{2}}(\gamma-1) \frac{\bar{p}^{\prime}}{\rho^{\prime}}\right]_{d}+\left[\frac{\theta \gamma}{(1-\theta \gamma)^{2}}(\gamma-1) \frac{\bar{\rho}^{\prime}}{\rho^{\prime}}\right]_{e}
$$

Then

$$
\begin{aligned}
{[]_{c}+\{\}_{\mathrm{IV}} } & =\frac{1}{(1-\theta \gamma)^{2}}\{\theta(1-\gamma)-(1-\theta \gamma)\}=-\frac{1-\theta}{(1-\theta \gamma)^{2}}, \\
{[]_{e}+\{\}_{\mathrm{III}} } & =\frac{1}{(1-\theta \gamma)^{2}}\{\theta \gamma(\gamma-1)+\theta \gamma(1-\theta \gamma)\} \frac{\bar{\rho}^{\prime}}{\rho^{\prime}} \\
& =\frac{1}{(1-\theta \gamma)^{2}}\left\{\theta \gamma^{2}-\theta^{2} \gamma^{2}\right\} \frac{\bar{\rho}^{\prime}}{\rho^{\prime}} \\
& =\frac{\theta \gamma^{2}(1-\theta)}{(1-\theta \gamma)^{2}} \frac{\bar{\rho}^{\prime}}{\rho^{\prime}} .
\end{aligned}
$$

Thus our formula for $\sigma$ is 


$$
\begin{aligned}
(1-\theta \gamma)^{2} \sigma= & \left\{\frac{1}{3} \frac{\theta}{A}\left[(1-3 z)(1-A) \gamma+2\left(1-\frac{A}{\theta}\right)\right]\right\}_{\mathrm{I}} \\
& +\theta(\gamma-1)^{2} \frac{\bar{p}^{\prime}}{\rho^{\prime}}+\theta(1-\theta) \gamma^{2} \frac{\bar{\rho}^{\prime}}{\rho^{\prime}}-(1-\theta),
\end{aligned}
$$

so that

$$
\begin{aligned}
(1-\theta \gamma)^{2} \sigma= & \left\{\frac{1}{3} \frac{\theta}{A}\left[(1-3 z)(1-A) \gamma+2\left(1-\frac{A}{\theta}\right)\right]\right\}_{\mathrm{I}} \\
& +\left\{\left[(\gamma-1)^{2}+\frac{\gamma^{2}(1-\theta)}{\bar{\sigma}}\right] \theta \frac{\overline{p^{\prime}}}{\rho^{\prime}}\right\}_{\mathrm{II}}-(1-\theta),
\end{aligned}
$$

where

$$
\frac{\bar{p}^{\prime}}{\rho^{\prime}}=\frac{1}{6} \frac{(1-A)}{A} \frac{(1+3 \bar{\mu} z)}{\gamma-1}
$$

This gives

$$
\begin{aligned}
(1-\theta \gamma)^{2} \sigma= & \frac{1}{3} \frac{\theta}{A}\left[(1-3 z)(1-A) \gamma+2\left(1-\frac{A}{\theta}\right)\right] \\
& +\frac{1}{6} \frac{\theta}{A}(1-A)(1+3 \bar{\mu} z)(\gamma-1)\left[1+\frac{\gamma^{2}(1-\theta)}{\bar{\sigma}(\gamma-1)^{2}}\right]+\theta-1
\end{aligned}
$$

By collecting terms, it is easily verified that (4.37) is equivalent to

$$
\begin{aligned}
(1-\theta \gamma)^{2} \sigma= & \frac{1}{6} \frac{\theta(1-A)}{A}[2(1-3 z) \gamma+(1+3 \bar{\mu} z)(\gamma-1)] \\
& +\frac{1}{6} \frac{\theta}{A}\left[(1+3 \bar{\mu} z)\left(\frac{\gamma^{2}}{\gamma-1}\right) \frac{1}{\bar{\sigma}}(1-\theta)(1-A)+4\right]+\theta-\frac{5}{3} .
\end{aligned}
$$

We use the following easily verifiable identities to eliminate $\gamma$ in favor of $z$ and then $z$ in favor of $\gamma$ in (4.38):

$$
\begin{aligned}
2(1-3 z) \gamma+(1+3 \bar{\mu} z)(\gamma-1) & =\frac{3-9 \bar{\mu} z^{2}-7 z+5 \bar{\mu} z}{z(1+\bar{\mu})}, \\
(1+3 \bar{\mu} z) \frac{\gamma^{2}}{\gamma-1} & =\frac{(1-3 \bar{\mu} z)(1+\bar{\mu} z)^{2}}{z(1-z)(1+\bar{\mu})} .
\end{aligned}
$$

Using (4.39) and (4.40) to eliminate $\gamma$ in favor of $z$ in (4.38) gives

$$
(1-\theta \gamma)^{2} \sigma=\frac{\theta(1-A)}{6 A(1+\bar{\mu})}\left\{\alpha(z, \bar{\mu})+\beta(z, \bar{\mu}) \frac{(1-\theta)}{\bar{\sigma}}\right\}+\frac{2}{3} \frac{\theta}{A}+\theta-\frac{5}{3},
$$

where

$$
\alpha \equiv \frac{3-7 z+5 \bar{\mu} z-9 \bar{\mu} z^{2}}{z}
$$




$$
\beta \equiv \frac{(1+3 \bar{\mu} z)(1+\bar{\mu} z)^{2}}{z(1-z)} .
$$

This gives $\sigma$, and completes the proof of Theorem 12 .

Alternatively, we can replace $z$ in favor of $\gamma$ in (4.38). By (4.41)-(4.43) we need only do the replacement in $\alpha$ and $\beta$. Since

$$
\gamma=\frac{\frac{1}{z}+\bar{\mu}}{1+\bar{\mu}}
$$

we have

$$
z=\frac{1}{(1+\bar{\mu}) \gamma-\bar{\mu}},
$$

and substituting this into (4.42) and (4.43) gives

$$
\begin{gathered}
\alpha=\frac{\left(3 \gamma^{2}+3 \bar{\mu} \gamma^{2}-\bar{\mu} \gamma-7 \gamma-2 \bar{\mu}\right)(1+\bar{\mu})}{(1+\bar{\mu}) \gamma-\bar{\mu}}, \\
\beta=\frac{(1+\bar{\mu}) \gamma+2 \bar{\mu}}{(1+\bar{\mu}) \gamma-\bar{\mu}} \frac{\gamma^{2}}{\gamma-1}(1+\bar{\mu}) .
\end{gathered}
$$

As a check, note that it is readily verified that (4.41) yields

$$
\sigma=\frac{\bar{\sigma}(\bar{\sigma}+7)}{3(1-\bar{\sigma})}
$$

when $z=\frac{1}{3}$, and $\bar{\mu}=\bar{\sigma}=$ const., and

$$
\theta=A=\frac{(1+\bar{\sigma})^{2}}{1+6 \bar{\sigma}+\bar{\sigma}^{2}}
$$

cf. [8].

As an application, we note that in light of (4.41), (3.30) and (3.53), the FRW-Lax shock condition holds at the shock-wave described by (3.22), (3.23) if and only if the following inequality holds (where $\alpha$ and $\beta$ are given by (4.42) and (4.43)):

$$
\frac{1}{6} \frac{\theta(1-A)}{A(1+\bar{\mu})}\left\{\alpha(z, \bar{\mu})+\beta(z, \bar{\mu}) \frac{(1-\theta)}{\bar{\sigma}}\right\}+\frac{2}{3} \frac{\theta}{A}+2 \theta>\frac{8}{3} .
$$

A direct consequence of (3.86)-(4.43) is Theorem 10 of Section 3, which states that, for outgoing shocks, the Lax characteristic conditions (3.53) and (3.54) hold, and $\sigma$, the sound speed squared, must be positive, if the shock is sufficiently strong. 


\section{Inadmissibility of $\bar{\rho}>\rho$}

In this section we prove the following theorem, which rules out $\bar{\rho}>\rho$ as physical, when $A>0$. (Recall that shocks with $p=p_{-}$(cf. (3.10)), have already been ruled out because then the shock speed is larger than the speed of light.)

Theorem 13. Assume that (2.20)-(2.22) hold. Then any shock wave determined by (3.22), (3.23) that satisfies $\bar{\rho}>\rho$ and $p=p_{+}$cannot have a positive sound speed $\sqrt{\sigma}$ because $\sigma<0$.

In order to prove this theorem, we need an alternative formula for $p^{\prime}$ (we always let "prime" denote $d / d \bar{r}$ ), which we now derive. Our strategy is to collect the coefficients of $\bar{p}^{\prime}, \bar{\rho}^{\prime}$, and $\rho^{\prime}$ in the expression for $p_{+}^{\prime}$, because these have a definite sign. We use the formulas

$$
\begin{gathered}
p_{+} \equiv p=\frac{\Theta \bar{\rho}-\rho}{1-\Theta}, \quad \Theta=\gamma \theta, \\
\gamma=\frac{\rho+\bar{p}}{\bar{\rho}+\bar{p}}, \quad \theta=\frac{A}{1-k r^{2}} .
\end{gathered}
$$

Differentiating $p$ with repect to $\bar{r}$ we get

$$
\begin{aligned}
(1-\Theta)^{2} p^{\prime} & =(1-\Theta)(\Theta \bar{\rho}-\rho)^{\prime}-(\Theta \bar{\rho}-\rho)(1-\Theta)^{\prime} \\
& =(1-\Theta)\left(\Theta^{\prime} \bar{\rho}+\Theta \bar{\rho}^{\prime}+\rho^{\prime}\right)-(\Theta \bar{\rho}-\rho)\left(-\Theta^{\prime}\right) \\
& =(\bar{\rho}-\rho) \Theta^{\prime}+(1-\Theta) \Theta \bar{\rho}^{\prime}-(1-\Theta) \rho^{\prime} ;
\end{aligned}
$$

thus

$$
(1-\Theta)^{2} p^{\prime}=(\bar{\rho}-\rho)\left(\theta^{\prime} \gamma+\theta \gamma^{\prime}\right)+(1-\Theta) \Theta \bar{\rho}^{\prime}-(1-\Theta) \rho^{\prime}
$$

Now

$$
\Theta^{\prime}=\frac{A^{\prime} \gamma}{1-k r^{2}}+\frac{A \gamma}{\left(1-k r^{2}\right)^{2}} 2 k r \frac{d r}{d \bar{r}}+\frac{A}{1-k r^{2}} \gamma^{\prime}
$$

or

$$
\Theta^{\prime}=\frac{A^{\prime} \gamma}{1-k r^{2}}+\frac{\theta \gamma}{\left(1-k r^{2}\right)} 2 k r \frac{d r}{d \bar{r}}+\theta \gamma^{\prime},
$$

and using (3.17) and (3.19) we get

$$
\frac{d r}{d \bar{r}}=\frac{\dot{r}}{\dot{\bar{r}}}=\frac{1}{\Theta R} .
$$

Using this and (4.11) in (5.3) gives 


$$
\Theta^{\prime}=\frac{8 \pi \mathscr{G} \gamma \bar{r}\left(\frac{1}{3} \rho-\bar{\rho}\right)}{1-k r^{2}}+\frac{2 k r}{\left(1-k r^{2}\right) R}+\theta \gamma^{\prime} .
$$

But

$$
\begin{aligned}
\gamma^{\prime}=\left(\frac{\rho+\bar{p}}{\bar{\rho}+\bar{p}}\right)^{\prime} & =\frac{(\bar{\rho}+\bar{p})\left(\rho^{\prime}+\bar{p}^{\prime}\right)-(\rho+\bar{p})\left(\bar{\rho}^{\prime}+\bar{p}^{\prime}\right)}{(\bar{\rho}+\bar{p})^{2}} \\
& =\frac{1}{(\bar{\rho}+\bar{p})} \rho^{\prime}-\frac{(\rho-\bar{\rho})}{(\bar{\rho}+\bar{p})^{2}} \bar{p}^{\prime}-\frac{(\rho+\bar{p})}{(\bar{\rho}+\bar{p})^{2}} \bar{\rho}^{\prime}
\end{aligned}
$$

so that

$$
\begin{aligned}
\Theta^{\prime}= & \frac{8 \pi \mathscr{G} \gamma \bar{r}\left(\frac{1}{3} \rho-\bar{\rho}\right)}{1-k r^{2}}+\frac{2 k r}{\left(1-k r^{2}\right) R}+\frac{\theta}{\bar{\rho}+\bar{p}} \rho^{\prime} \\
& -\frac{\theta(\rho-\bar{\rho})}{(\bar{\rho}+\bar{p})^{2}} \bar{p}^{\prime}-\frac{\theta(\rho+\bar{p})}{(\bar{\rho}+\bar{p})^{2}} \bar{\rho}^{\prime} .
\end{aligned}
$$

Using (5.6) we get

$$
\begin{aligned}
(1-\Theta)^{2} p^{\prime}= & (\bar{\rho}-\rho) \Theta^{\prime}+(1-\Theta) \Theta \bar{\rho}^{\prime}-(1-\Theta) \rho^{\prime} \\
= & \frac{(\bar{\rho}-\rho)}{\left(1-k r^{2}\right) \bar{r}}\left\{8 \pi \mathscr{G} \gamma\left(\frac{1}{3} \rho-\bar{\rho}\right) \bar{r}^{2}+2 k r^{2}\right\} \\
& +\left\{\frac{\theta(\rho-\bar{\rho})^{2}}{(\bar{\rho}+\bar{p})^{2}}\right\}_{\mathrm{I}} \bar{p}^{\prime}+\left\{-(1-\Theta)+\frac{(\bar{\rho}-\rho) \theta}{(\bar{\rho}-\bar{p})}\right\}_{\mathrm{II}} \rho^{\prime} \\
& +\left\{(1-\Theta) \Theta-\frac{(\rho+\bar{p})(\bar{\rho}-\rho) \theta}{(\bar{\rho}+\bar{p})^{2}}\right\}_{\mathrm{III}} \bar{\rho}^{\prime} .
\end{aligned}
$$

But

$$
\begin{gathered}
\{\}_{\mathrm{III}}=\Theta\left\{1-\Theta-\frac{\bar{\rho}-\rho}{\bar{\rho}+\bar{p}}\right\} \\
=\Theta\left\{1-\frac{(\rho+\bar{p})}{(\bar{\rho}+\bar{p})} \theta-\frac{(\bar{\rho}-\rho)}{(\bar{\rho}+\bar{p})}\right\} \\
=\Theta \frac{(\rho+\bar{p})(1-\theta)}{(\bar{\rho}+\bar{p})}=\Theta \gamma(1-\theta), \\
\{\}_{\mathrm{II}}=-1+\frac{(\rho+\bar{p}) \theta}{(\bar{\rho}+\bar{p})}+\frac{(\bar{\rho}-\rho) \theta}{(\bar{\rho}+\bar{p})}=-1+\frac{(\bar{\rho}+\bar{p}) \theta}{(\bar{\rho}+\bar{p})}=-1+\theta,
\end{gathered}
$$

so that (5.7) becomes

$$
\begin{aligned}
(1-\Theta)^{2} p^{\prime}= & \frac{(\bar{\rho}-\rho)}{\left(1-k r^{2}\right) \bar{r}}\left\{8 \pi \mathscr{G} \gamma\left(\frac{\rho}{3}-\bar{\rho}\right) \bar{r}^{2}+2 k r^{2}\right\}_{\mathrm{IV}} \\
& +\frac{\theta(\bar{\rho}-\rho)^{2}}{(\bar{\rho}+\bar{p})^{2}} \bar{p}^{\prime}+\gamma(1-\theta) \Theta \bar{\rho}^{\prime}-(1-\theta) \rho^{\prime} .
\end{aligned}
$$


Now using (2.20) and (2.21), together with the Oppenheimer-Volkoff equation (2.15), we see that $\bar{p}^{\prime}$ and $\bar{\rho}^{\prime}$ are both negative. Furthermore, from (2.37), $\rho^{\prime}>0$ because $\bar{\rho}>\rho$. Thus (5.9) implies that $p^{\prime}<0$ provided that \{\}$_{\mathrm{IV}}<0$. Now

$$
\{\}_{\mathrm{IV}}=8 \pi \mathscr{G}\left(\frac{1}{3}-\frac{\bar{\rho}}{\rho}\right) \gamma \rho \bar{r}^{2}+2 k r^{2}
$$

so using (4.17) and (4.19) we have

$$
\{\}_{\mathrm{IV}}=(1-A)\left\{\left(1-3 \frac{\bar{\rho}}{\rho}\right) \gamma+2 \frac{1-\frac{A}{\theta}}{1-A}\right\} .
$$

Since $z=\bar{\rho} / \rho$ and $\bar{\mu}=\bar{p} / \bar{\rho}$, we have

$$
\gamma=\frac{\frac{1}{z}+\bar{\mu}}{1+\bar{\mu}}
$$

so that

$$
\{\}_{\mathrm{IV}}=\frac{(1-A)}{1+\bar{\mu}}\left\{(1-3 z)\left(\frac{1}{z}+\bar{\mu}\right)+2(1+\bar{\mu}) \frac{1-\frac{A}{\theta}}{1-A}\right\} .
$$

But the largest the fraction

$$
\frac{1-\frac{A}{\theta}}{1-A}
$$

can be for fixed $A$ is when $\theta=1$, so that

$$
\frac{1-\frac{A}{\theta}}{1-A} \leqq 1 \text {. }
$$

Thus for \{\}$_{\text {IV }}<0$, it suffices to show that

$$
f(z) \equiv(1-3 z)\left(\frac{1}{z}+\bar{\mu}\right)+2(1+\bar{\mu}) \leqq 0
$$

for $z \geqq 1$. Now $f(1)=0$, and

$$
f^{\prime}(z)=-3\left(\frac{1}{z}+\bar{\mu}\right)+(1-3 z)\left(-\frac{1}{z^{2}}\right)<0
$$

for $z \geqq 1$, and so

$$
f(z) \leqq 0
$$

for $z \geqq 1$. Thus \{\}$_{\mathrm{IV}}<0$ for $z>1$. Therefore $p^{\prime}<0$ when $\bar{\rho}>\rho$, and as $\rho^{\prime}>0$, this proves that the sound speed $\sigma=p^{\prime} / \rho^{\prime}<0$. 
Acknowledgement. The research of J. SMOLLER was supported in part by NSF Applied Mathematics Grant Number DMS-92-06631, in part by ONR, US NAVY grant number N00014-94-1-0691 and by the Institute of Theoretical Dynamics, UC-Davis. The research of B. TEMPLE was supported in part by NSF Applied Mathematics Grant Number DMS-92-06631, in part by ONR, US NAVY grant number N00014-94-1-0691, a Guggenheim Fellowship, and by the Institute of Theoretical Dynamics, UC-Davis.

\section{References}

[1] W. IsRAeL, Singular hypersurfaces and thin shells in general relativity, Il Nuovo Cimento, 44 B (1966), 1-14.

[2] P. D. Lax, Hyperbolic systems of conservation laws, II, Comm. Pure Appl. Math., 10 (1957), 537-566.

[3] J. R. OPPENHEIMER \& J. R. SNYder, On continued gravitational contraction, Phys. Rev., 56 (1939), 455-459.

[4] J. Smoller, Shock-Waves and Reaction-Diffusion Equations, Second edn., Springer-Verlag, 1994.

[5] J. Smoller, B. Temple \& Z. P. Xin, Instability of rarefaction shocks for systems of conservation laws, Arch. Rational Mech. Anal., 112, (1990), 63-81.

[6] J. Smoller \& B. Temple, Global solutions of the relativistic Euler equations, Comm. Math. Phys., 157 (1993), 67-99.

[7] J. SMOller \& B. Temple, Shock-wave solutions of the Einstein equations: The Oppenheimer-Snyder model of gravitational collapse extended to the case of nonzero pressure, Arch. Rational Mech. Anal., 128 (1994), 249-297.

[8] J. Smoller \& B. Temple, Astrophysical shock-wave solutions of the Einstein equations, Phys. Rev. D, 51 (1995), 2733-2743.

[9] R. Tolman, Static solutions of Einstein's field equations for spheres of fluid, Phys. Rev., 55 (1939), 364-374.

[10] R. Tolman, Relativity, Thermodynamics and Cosmology, Oxford University Press, 1934.

[11] S. Weinberg, Gravitation and Cosmology: Principles and Applications of the General Theory of Relativity, Wiley, New York, 1972.

Department of Mathematics University of Michigan

Ann Arbor, Michigan 48109

and

Department of Mathematics University of California Davis, California 95616

(Accepted January 19, 1996) 